\title{
A Practical and Consistent Parametrization of Dark Matter Self-Interactions
}

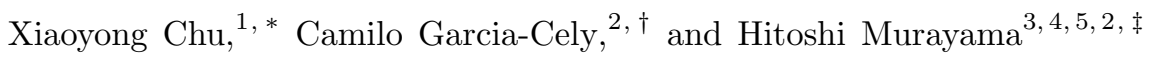 \\ ${ }^{1}$ Institute of High Energy Physics, Austrian Academy of Sciences, Nikolsdorfer Gasse 18, 1050 Vienna, Austria \\ ${ }^{2}$ Deutsches Elektronen-Synchrotron DESY, Notkestrasse 85, 22607 Hamburg, Germany \\ ${ }^{3}$ Department of Physics, University of California, Berkeley, CA 94720, USA \\ ${ }^{4}$ Kavli Institute for the Physics and Mathematics of the Universe (WPI), University of Tokyo, Kashiwa 277-8583, Japan \\ ${ }^{5}$ Ernest Orlando Lawrence Berkeley National Laboratory, Berkeley, CA 94720, USA
}

\begin{abstract}
Self-interacting dark matter has been proposed to explain the apparent mass deficit in astrophysical small-scale halos, while observations from galaxy clusters suggest that the corresponding cross section depends on the velocity. Accounting for this is often believed to be highly model-dependent with studies mostly focusing on scenarios with light mediators. Based on the effective-range formalism, in this work we point out a model-independent approach which accurately approximates the velocity dependence of the self-interaction cross section with only two parameters. We illustrate how this parameterization can be simultaneously interpreted in various well-motivated scenarios, including self-interactions induced by Yukawa forces, Breit-Wigner resonances and bound states. We investigate the astrophysical implications and discuss how the approximation can be improved in certain special regimes where it works poorly.
\end{abstract}

\section{Contents}

\section{Introduction}

\section{The effective-range formalism}

III. Astrophysical implications

A. Velocity dependence in effective range theories 5

B. Realistic velocity distributions

IV. Interpreting $a$ and $r_{e}$ in terms of model parameters

A. Contact interaction

B. SIDM with a light mediator

C. SIDM via bound states or virtual levels

D. Resonant SIDM

E. SIMPs

\section{Improving the effective-range} approximation
A. Antiresonances
B. Sharp resonances
C. Inelastic scatterings

\section{Summary and Outlook}

A. The effective range theory

1. A simple method to find the phase shift

2. The $S$-wave case

3. The Hulthén Potential

\section{B. Velocity-averaged cross sections}

\footnotetext{
*Electronic address: xiaoyong.chu@oeaw.ac.at

${ }^{\dagger}$ Electronic address: camilo.garcia.cely@desy.de

${ }^{\ddagger}$ Electronic address: hitoshi@berkeley.edu, hitoshi.murayama@ipm i.jp Hamamatsu Professor
}

\section{INTRODUCTION}

In contrast to the ordinary substances found on Earth, more than three quarters of the matter in the Universe is not made of protons, neutrons, or electrons. This is the so-called dark matter (DM) and identifying its particle nature is one of the chief goals of particle physics and cosmology today. According to the $\Lambda \mathrm{CDM}$ model [1, which accurately describes the Universe at cosmological scales, DM interacts very weakly with normal matter and it was cold and collisionless during the formation of struc8 tures in the early universe. Although DM can be treated as collisionless particles at large scales, non-gravitational DM scatterings can still occur in the dense central regions of small-scale halos such as those of dwarf or lowsurface-brightness galaxies. This is the self-interacting dark matter (SIDM) hypothesis, which was proposed [2] to explain the seeming discrepancies between observations of the smallest DM halos that we can currently observe and certain predictions of the $\Lambda \mathrm{CDM}$ model; see [3, 4] for recent reviews.

The aforementioned discrepancies can be explained if DM elastically scatters with a cross section per unit of mass as large as several $\mathrm{cm}^{2} / \mathrm{g}$ when it moves at approximately $10 \mathrm{~km} / \mathrm{s}$, i.e., roughly the $\mathrm{DM}$ velocity dispersion in small-scale objects [5-10]. Meanwhile, recent studies on halo dynamics at cluster scales provide upper bounds on the self-interaction cross section of around $0.2-1 \mathrm{~cm}^{2} / \mathrm{g}$ [11-16], which are associated with typical DM velocities of the order of $10^{3} \mathrm{~km} / \mathrm{s}$.

A natural question then arises: how can these observations be interpreted in terms of the properties of the DM particle? One possibility is to postulate a specific DM model, and translate the previous velocity-dependent cross section in terms of masses and couplings. For instance, this has been done for scenarios where DM interacts by means of a light mediator [17-19] and for models in which DM resonantly self-scatters [20 22]. Neverthe- 
less, from the phenomenological point of view, this is not very practical, not only because the cross sections typically depend in a complicated way on the model parameters, but also because there are a myriad of SIDM scenarios. The essence of this work is to propose a simple parametrization of the DM self-interaction cross section, which approximates with great accuracy the velocitydependent effects, and most importantly, which interpolates the predictions of different DM scenarios allowing to establish comparisons among them. More precisely, here we advocate the use of the effective-range theory as a model-independent way to study the velocity dependence of SIDM. Notice that this approach has been adopted in concrete models of DM before [23 27].

The effective-range approach was formulated [28, 29] as an effort to explain the non-relativistic scattering of neutrons by protons. Based on simple assumptions from quantum mechanics, this approach suggests that the scattering observables can be parametrized in terms of two quantities: the scattering length $a$, and the effective range $r_{e}$. While being very predictive, the effective-range theory does not demand a precise knowledge of the underlying interactions among the colliding particles, apart from the requirement that the scattering force must vanish at sufficiently large distances. In fact, due to this, it can describe the non-relativistic scattering induced by contact interactions, light mediators, and Breit-Wigner resonances, among others.

A brief historical remark may be helpful to readers. ${ }^{1}$ In late 1940s people proposed different models to describe nucleon-nucleon scattering cross sections at low energies. After many explicit calculations, it became clear that only two parameters are relevant, independent of details of models. Schwinger came up with a proof why that was the case in an unpublished lecture note. Blatt and Jackson 29] showed with more calculations that indeed only two parameters were necessary to explain the data. Then Bethe 28 came up with a simple and elegant proof (reproduced in Appendix A to understand this observation. The flip side of this remarkable simplicity is that we gain very little information on the detailed model from the data. Bethe wrote "practically no information could be obtained, from classical scattering experiments, on the shape of the potential." On the other hand, for the purpose of describing the impact of self-interaction among dark matter particles in various halos, this is a boon; we need to specify only two parameters (and the mass of dark matter) in order to simulate the impact of selfinteractions without the need for dealing with explicit models. This is why we propose the use of the effective range theory for the study of SIDM.

This paper is organized as follows. In Section II, we review the effective-range approach, and explain how it can

\footnotetext{
${ }^{1}$ Hans Bethe personally described the history in a YouTube video
} https : //www . youtube. com/watch?v=hbcQMG2XpTI. be useful to describe DM self-scattering. In Section III, we discuss the implications of this approach for describing the velocity-dependence of DM self-interactions in astrophysical halos in a model-independent way. In Section IV, we investigate how to relate the scattering length and effective range to parameters of concrete DM models. In Section V, we propose a concrete method on how the effective-range approach can be extended or improved in some cases where it fails. Section VI provides the final conclusions and future prospects.

\section{THE EFFECTIVE-RANGE FORMALISM}

Before focusing on SIDM, we will first introduce the effective-range approximation. In any collision the differential cross section $d \sigma / d \Omega$ determines the scattering rate and describes the velocity dependence of the process. Up to a possible symmetry factor, it is given by $d \sigma=|f(k, \theta)|^{2} d \Omega$, where $f(k, \theta)$ is the scattering amplitude with $k$ being the incoming momentum. ${ }^{2}$ For collisions with definite orbital angular momentum, $\ell$, the amplitude is proportional to the Legendre polynomial, $P_{\ell}(\cos \theta)$, with the corresponding coefficient defining the partial-wave amplitude, $f_{\ell}(k)$. More precisely

$$
\begin{aligned}
& f(k, \theta)=\sum_{\ell=0}^{\infty}(2 l+1) f_{\ell}(k) P_{\ell}(\cos \theta) \\
& \text { with } f_{\ell}(k) \equiv \frac{e^{2 i \delta_{\ell}(k)}-1}{2 i k}=\frac{1}{k\left(\cot \delta_{\ell}(k)-i\right)} .
\end{aligned}
$$

The second relation defines the phase shift, $\delta_{\ell}(k)$ for the $\ell$ partial wave. While the precise value of $\delta_{\ell}(k)$ must be obtained by solving the Schrödinger equation describing the scattering process, this phase shift always satisfies some general requirements. For instance, it must be real if inelastic processes are absent. In this work, we are concerned with elastic scatterings in astrophysical halos, as a result, unless stated otherwise, we will assume that inelastic processes are relatively weaker and take $\delta_{\ell}$ real (see Section VC for how to include the inelastic processes).

Another requirement on the phase shift is that, for finite-range interactions, the function $k^{2 \ell+1} \cot \delta_{\ell}(k)$ must be analytic at $k=0$ (for more details see Appendix Ap. The effective-range approximation [28, 29] consists in neglecting the high-order terms in the cor-

\footnotetext{
${ }^{2}$ Here and below, we separate the center-of-mass motion and hence $k$ is the relative momentum of two particles that scatter. The orbital angular momentum $\ell$ below is defined in the center-ofmass frame. Note also that we are dealing with low velocities, less than about $10^{-2} c$, which are typical in halos, so the use of non-relativistic quantum mechanics is justified.
} 

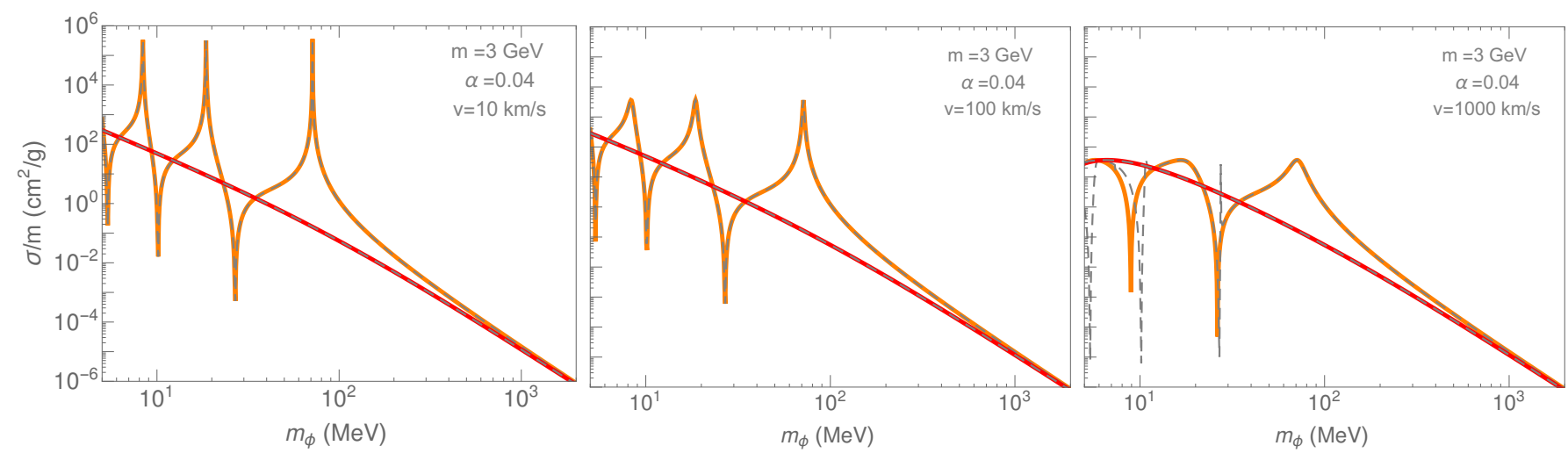

Figure 1: Comparison of the numerical S-wave cross section per unit mass (solid) against the effective-range approximation (dashed). Orange and red lines correspond to the numerical results for attractive and repulsive forces, respectively. Note that the mass here is the actual mass of the scattering particles, not the reduced mass.

responding expansion in $k^{2}$, so that

$$
k^{2 \ell+1} \cot \delta_{\ell}(k) \simeq-\frac{1}{a_{\ell}^{2 \ell+1}}+\frac{1}{2 r_{e, \ell}^{2 \ell-1}} k^{2} .
$$

The quantities $a_{\ell}$ and $r_{e, \ell}$ thus defined are known as the scattering length and the effective range, respectively. This approximation describes the phase shift with good accuracy at sufficiently low energies. Consequently, if one partial wave dominates the scattering process, the velocity dependence of the cross section is determined by only two parameters.

Let us focus on the $S$-wave case, which dominates the low-energy scattering rate in many situations of interest. In this case, the cross section is given by ${ }^{3}$

$$
\sigma_{0}=\frac{4 \pi}{k^{2}} \sin ^{2} \delta_{0} \approx \frac{4 \pi a^{2}}{1+k^{2}\left(a^{2}-a r_{e}\right)+\frac{1}{4} a^{2} r_{e}^{2} k^{4}} .
$$

Note that the unitarity bound, $4 \pi / k^{2}$, is saturated for $|a| \rightarrow \infty$. One important example of this kind is the case of particles of mass $m$ interacting via the Yukawa potential

$$
V(r)= \pm \alpha \frac{e^{-m_{\phi} r}}{r}
$$

Fig. 1 compares the numerically-evaluated cross section and the approximation based on Eq. (4) for a particular region of the parameter space. ${ }^{4}$ Despite its simplicity, the effective range approximation works very well, i.e.

\footnotetext{
${ }^{3}$ From now on, for simplicity we will omit the subscript $\ell=0$ for the scattering length and the effective range in the $S$-wave.

${ }^{4}$ Note that it is essential to go beyond the lowest-order perturbation theory (first Born approximation) $\sigma=4 \pi\left(\alpha m / m_{\phi}^{2}\right)^{2}$, which does not depend on the sign of $\alpha$ nor produces spikes in the full calculation. Effective range theory reproduces both correctly.
}

high-order terms of $k^{2}$ in Eq. (3) can be neglected. In particular, it is able to reproduce the peak structure of the cross section. As is well known, such peaks are related to zero-energy bound states induced by the attractive potential.

A similar example is the non-relativistic scattering of two nucleons. In the case of proton-neutron collision, for the spin-one channel and kinetic energies up to a few $\mathrm{MeV}$, Eq. (4) accurately describes the velocity dependence of the corresponding cross section with $a=5.42 \mathrm{fm}$ and $r_{e}=1.75 \mathrm{fm}$ (see e.g. [30, 31]). This is also related to a bound state: the deuteron.

Likewise, the collision of two neutrons -for which the total spin is zero- can be characterized by $a=-18.9 \mathrm{fm}$ and $r_{e}=2.75 \mathrm{fm}$. In contrast, in this case no real bound state exists. Instead, the scattering is induced by a virtual level, ${ }^{5}$ commonly known as the dineutron (see Fig. 2p.

Equally interesting is the fact that Breit-Wigner resonances can also be described using the effective-range approximation. For simplicity let us suppose that the colliding particles are scalars with the same mass $m$, and that the resonance has spin $\ell$. Thus, if the energy, $E=k^{2} / m$, is sufficiently close to the resonance $E_{R}$, the cross section

\footnotetext{
${ }^{5}$ A bound state is a pole in the scattering amplitude $f_{\ell}(k)$ along the positive imaginary axis on the complex $k$ plane, which corresponds to exponential damping of the radial wave function $\propto e^{i k r}$. When parameters of the potential are varied, a pole may move to the negative imaginary axis, which no longer describes a bound state because the wave function grows exponentially. However, the existence of a pole in the scattering amplitude can produce a pronounced enhancement in the cross section and is hence important. In this case, the pole is called a virtual level. It should not be confused with virtual particles or virtual states that refer to intermediate particles or states (propagators) in perturbation theory.
} 


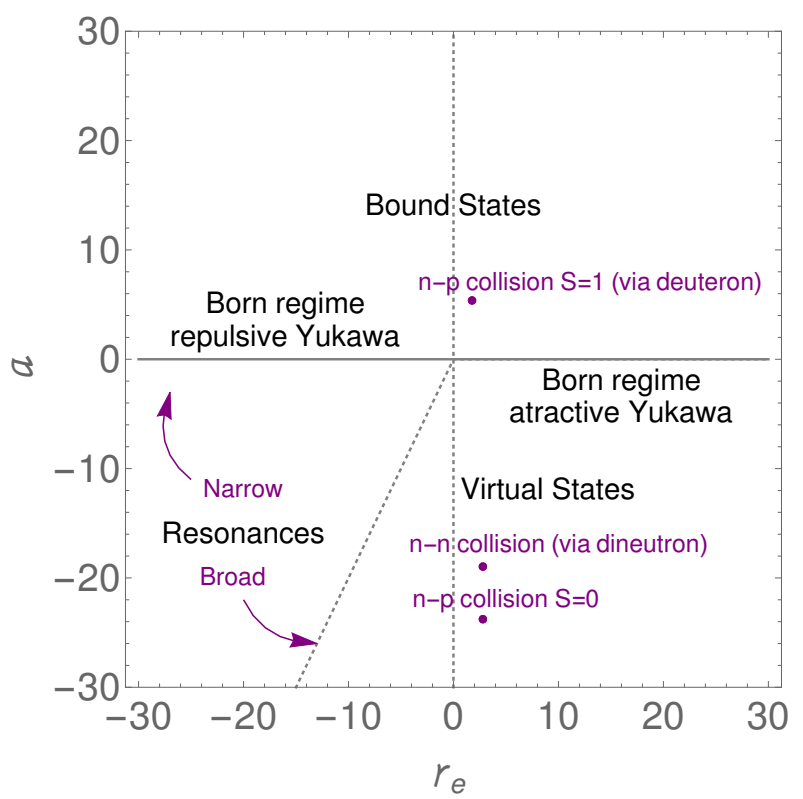

Figure 2: Sketch of the scattering length and the effective range, which determine the cross section using Eq. (4). They simultaneously parametrize the non-relativistic scattering in seemingly different theories including that induced by a Yukawa force, collisions via Breit-Wigner resonances, scatterings induced by bound state or virtual level, and the collision of non-relativistic protons (p) and neutrons (n). For the last case, the displayed values in units of fm accurately describe the experimental data 31.

is dominated by the partial wave $\ell$ so that

$$
\sigma_{\ell}=\frac{4 \pi(2 l+1)}{m E} \frac{\Gamma(E)^{2} / 4}{\left(E-E_{R}\right)^{2}+\Gamma^{2}(E) / 4} .
$$

The width in general varies with the energy in such way that $\Gamma(E) \propto E^{(2 \ell+1) / 2}$ (see e.g. 20]). Using the effectiverange approximation to the phase shift (Eq. (3p), we find that the cross section, $\sigma_{\ell}=4 \pi(2 \ell+1) \sin ^{2} \delta_{\ell} / k^{2}$, exactly matches the previous formula with

$$
a_{\ell}=-\frac{\Gamma\left(E_{R}\right)^{\frac{1}{2 \ell+1}}}{2^{\frac{1}{2 \ell+1}} E_{R}^{\frac{2 \ell+3}{4 \ell+2}} m^{\frac{1}{2}}}, \quad r_{e, \ell}=-\frac{2^{\frac{2}{-2 \ell+1}} E_{R}^{\frac{2 \ell+1}{-4 \ell+2}}}{\Gamma^{\frac{1}{-2 \ell+1}} m^{\frac{1}{2}}} .
$$

Far from the resonance, some deviations are expected. In fact, as shown in right panel of Fig. 11 the effectiverange approximation also fails close to the antiresonances, i.e., where the cross section vanishes. We will elaborate more on these cases in Section $\mathrm{V}$. Likewise, when the range of the Yukawa potential, $m_{\phi}^{-1}$, is close to or larger than the de Broglie wavelength of the incoming particles, $k^{-1}$, the approximation fails. This region, usually referred to as classical regime, corresponds to $m_{\phi} \lesssim 5 \mathrm{MeV}$ for the parameter region of the right panel of Fig. 1. In fact, this is true for any potential, for

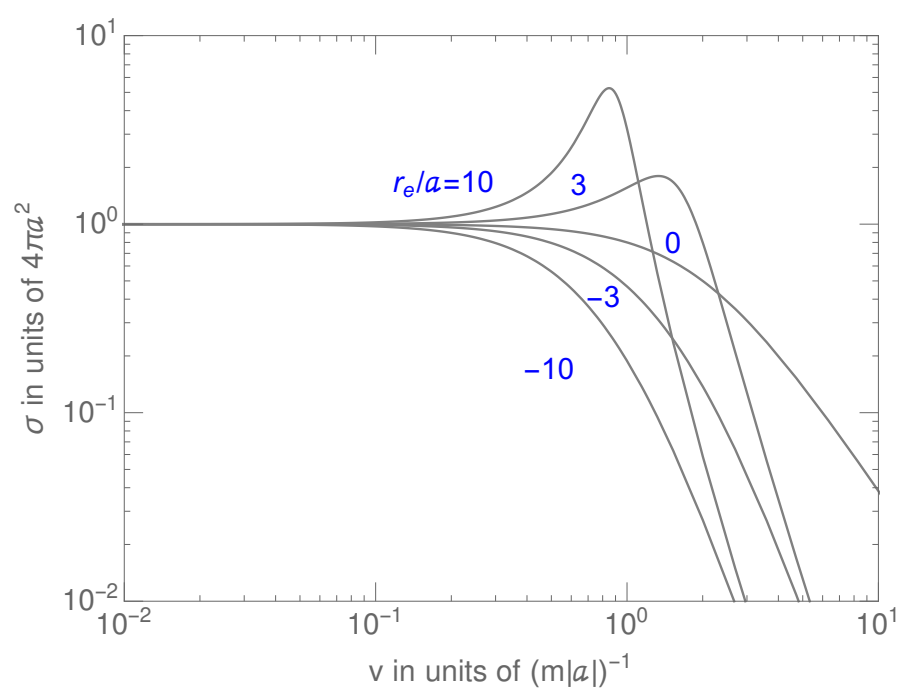

Figure 3: Self-scattering cross section as a function of the velocity for the indicated ratios of the effective range to scattering length.

momenta larger than the inverse of the force range, not only must one include higher-order terms in Eq. (3), but also the differential cross section receives contributions from high partial waves. In this case, the exact values of more phase shifts $\delta_{\ell}$ are needed to obtain the total scattering cross section. As a result, the effective-range approach can not be applied for long-range forces. For more details, see e.g. 32 34.

In summary, the effective-range approximation properly describes many types of low-energy scattering. These cases differ in the magnitude and sign of the effective range parameters, as will be explained in Section IV and as sketched in Fig. 2 .

\section{ASTROPHYSICAL IMPLICATIONS}

The main hypothesis of SIDM paradigm is that smallscale DM halos such as those of dwarf galaxies do not develop a high central density because its DM particles self-scatter with a cross section per unit mass in the range $\left.1-10 \mathrm{~cm}^{2} / \mathrm{g} 6\right]$. On the other hand, observations of clusters of galaxies indicate that $\sigma / m \lesssim$ $0.2-1 \mathrm{~cm}^{2} / \mathrm{g}$ [11 16]. Since in the former objects the average DM relative velocity is typically of the order of 10 $\mathrm{km} / \mathrm{s}$, whereas in clusters of galaxies it is around 2000 $\mathrm{km} / \mathrm{s}$, a velocity-dependent cross section is required in order to accommodate both.

Before discussing this in detail, let us note that we use $m$ for the DM mass, $v$ for the relative velocity between two initial DM particles in the centre-of-mass (CM) frame, $m_{\star}=m / 2$ for the reduced mass and $k=m_{\star} v=m v / 2$ for each incoming DM momentum. 



Figure 4: Contours of the cross section per unit of mass at cluster scales $(v \sim 2000 \mathrm{~km} / \mathrm{s})$ for the indicated cross sections at zero velocity. In the parameter space shown in each plot, the latter coincides within $1 \%$ with the cross section at dwarf-galaxy scales $(v \sim 10 \mathrm{~km} / \mathrm{s})$. The gray area, where $\sigma / m \gtrsim 1 \mathrm{~cm}^{2} / \mathrm{g}$, is excluded by cluster observations [11]13.

\section{A. Velocity dependence in effective range theories}

In the effective-range framework, the velocitydependent cross section is given by ${ }^{6}$

$$
\sigma(v)=4 \pi a^{2}\left(\left(1-\frac{1}{8} \frac{r_{e}}{a}(m a v)^{2}\right)^{2}+\frac{1}{4}(m a v)^{2}\right)^{-1},
$$

where the signs of the scattering length and the effective range only enter in the equation via their ratio, and can not be separately constrained by studying the velocity dependence of the DM scattering.

The velocity-dependence of the scattering cross section is also shown in Fig. 3. At very low velocities the cross section is roughly constant and equal to $4 \pi a^{2}$. If $r_{e} / a<1$, the cross section monotonously decreases, most appreciably for high DM velocities, $v \gtrsim(m|a|)^{-1}$. In contrast, if $r_{e} / a>1$, the cross section increases with $v$ until it reaches the maximum $4 \pi r_{e}^{2} a /\left(2 r_{e}-a\right)$ at $v_{\text {peak }}=2\left(m\left|r_{e}\right|\right)^{-1} \sqrt{2\left(r_{e} / a-1\right)}$ and then decreases. If $|a| \ll 1 / m$, the corresponding cross section can be con-

\footnotetext{
${ }^{6}$ The transfer cross section, $\sigma_{T}=\int d \sigma(1-\cos \theta)$, is typically used as a proxy for the scattering effects in DM halos. This is because, on the one hand, $\sigma_{T}$ takes into account that perpendicular scattering is most efficient for thermalizing the DM halo and affecting structure observables. On the other hand, SIDM studies often discuss scatterings induced by the exchange of a light mediator, which exhibits a divergence in the forward direction regularized by the transfer cross section. For $S$-wave scattering, $\sigma_{T}$ and $\sigma_{0}$ coincide and are therefore interchangeable.
}

sidered as a constant in all realistic DM halos.

To numerically illustrate this for halos of various sizes, Fig. 4 shows the contours of the self-interaction cross section per unit mass at $v=2000 \mathrm{~km} / \mathrm{s}$ for $\sigma /\left.m\right|_{v \rightarrow 0}$ equal to $1 \mathrm{~cm}^{2} / \mathrm{g}$ (left) and $10 \mathrm{~cm}^{2} / \mathrm{g}$ (right). We would like to note that $\sigma /\left.m\right|_{v \rightarrow 0}$ approximates the corresponding values in dwarf scales at $1 \%$ level in the parameter space shown in the figure. From Fig. 4, we conclude that GeV SIDM is associated with scattering lengths of several fm and that sub-GeV SIDM is either excluded by cluster observations or requires a cross section of around $1 \mathrm{~cm}^{2} / \mathrm{g}$ throughout all scales of interest. While some of these conclusions have been obtained in specific SIDM scenarios such as those involving a light mediator [35] or resonant SIDM [20], we would like to emphasize that these conclusions apply to any model where the effective-range approach applies.

Fig. 4 also suggests that the ratio $r_{e} / a$ is poorly constrained by the velocity dependence of the cross section. In the light of this and in order to consider a wider range of parameter space, in Fig. 5 we show three possibilities for the effective range compared to the scattering length. Concretely, for each fixed value of $r_{e} / a$, we illustrate the parameter space simultaneously satisfying

$$
\begin{aligned}
& 1 \mathrm{~cm}^{2} / \mathrm{g}\left.\lesssim \frac{\sigma}{m}\right|_{v=10 \mathrm{~km} / \mathrm{s}} \lesssim 10 \mathrm{~cm}^{2} / \mathrm{g}, \\
&\left.\frac{\sigma}{m}\right|_{v=2000 \mathrm{~km} / \mathrm{s}} \lesssim 1 \mathrm{~cm}^{2} / \mathrm{g} .
\end{aligned}
$$

The former takes place within the colored region while 

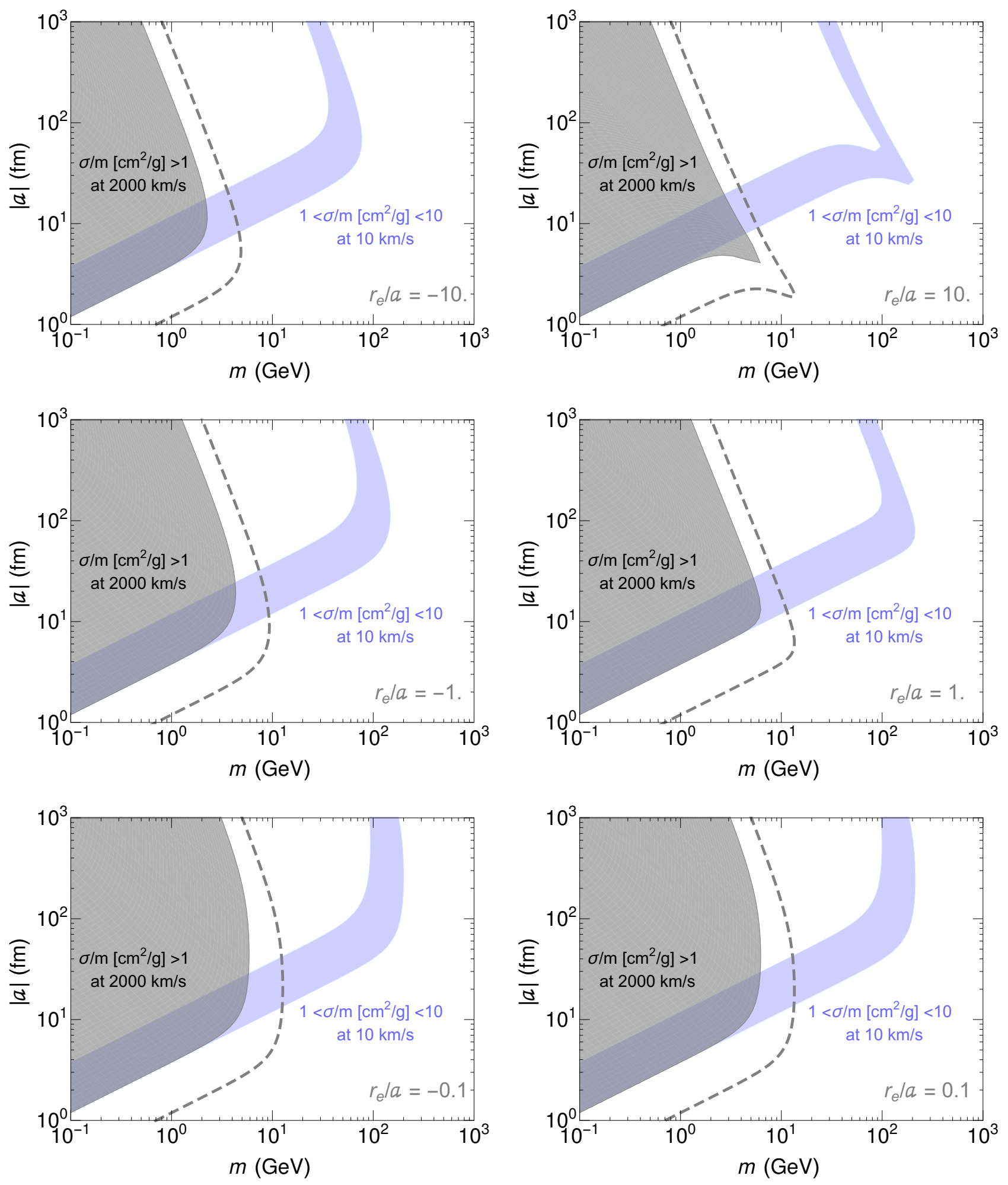

Figure 5: Contours of $\sigma / m$ within the range of $1 \mathrm{~cm}^{2} / \mathrm{g}-10 \mathrm{~cm}^{2} / \mathrm{g}$ at dwarf scales $(v=10 \mathrm{~km} / \mathrm{s})$. The gray areas represent the exclusion limit from cluster-scale observables $(v=2000 \mathrm{~km} / \mathrm{s})$, and are extended to the gray dashed curves if one requires $\sigma / m \lesssim 0.2 \mathrm{~cm}^{2} / \mathrm{g}$ at cluster scales. 
the latter, as in Fig. 4 corresponds to the region not excluded by the gray area. Recent studies have claimed stronger constraints of $0.2 \mathrm{~cm}^{2} / \mathrm{g}$ from observations of galaxy clusters [14 16, which are indicated as a gray dashed line in each panel.

In each panel there are regions where all the constraints are simultaneously satisfied. This confirms our previous remark that the ratio $r_{e} / a$ is largely unconstrained. Likewise, scenarios with a cross section of $1 \mathrm{~cm}^{2} / \mathrm{g}$ at dwarf and cluster scales are those for which the borders of the gray and the colored regions lie on top of each other and -as mentioned above- they correspond to DM masses below a few $\mathrm{GeV}$.

\section{B. Realistic velocity distributions}

So far we have assumed a monochromatic velocity distribution for all DM particles in each halo. Below, we take into account the realistic distribution of DM velocities and then consider the corresponding average cross section for individual DM halos. The former is typically achieved by assuming a Maxwell-Boltzmann distribution with a cut-off scale:

$$
f\left(v, v_{0}\right)=\frac{4 v^{2} e^{-v^{2} / v_{0}^{2}}}{\sqrt{\pi} v_{0}^{3}} \Theta\left(v_{\max }-v\right),
$$

where $v_{\max }$ is the escape velocity and $v_{0}$ is a parameter determining the typical velocities in the DM halo. For $v_{\max } \gg v_{0}$, the average velocity is $\langle v\rangle=2 v_{0} / \sqrt{\pi}$. More concretely, we take the average cross section $\langle\sigma\rangle \equiv$ $\left(\int_{0}^{\infty} f\left(v, v_{0}\right) \sigma v d v\right) /\left(\int_{0}^{\infty} f\left(v, v_{0}\right) v d v\right)$, as the exact value of $v_{\max }$ has only little effect in practice. Using a MaxwellBoltzmann distribution would at most modify the curves of Fig. 5 mildly, so the conclusion of the previous subsection remains unchanged. For a detailed calculation and a numerical comparison of $\langle\sigma v\rangle$ and $\sigma(\langle v\rangle)\langle v\rangle$, see Appendix. B.

On the observational side, extracting cross sections from experimental data is challenging and generally requires delicate N-body simulations at present. An intermediate method is given by the semi-analytical method proposed in [12], which allows to infer the velocityaveraged cross section per unit mass, $\langle\sigma v\rangle / m$, for a given DM halo. This method was applied to five clusters from [36], seven low-surface-brightness (LSB) spiral galaxies in [37] and six dwarf galaxies of the THINGS sample 38] (see also 39]). Fig. 6 shows their resulting values in green, blue and red, respectively.

Using the velocity-averaged scattering cross section, we can fit $|a|, r_{e} / a$ and $m$ to the aforementioned semianalytical results and thus constrain the parameters of the effective-range theory. The best-fit point is shown in

\begin{tabular}{lllll}
\hline & S1 & S2 & S3 & S4 \\
\hline$a(\mathrm{fm})$ & 19.2 & 25.6 & 3.8 & 37.4 \\
$r_{e}(\mathrm{fm})$ & 0.01 & 256.1 & -57. & -748.9 \\
$m(\mathrm{GeV})$ & 14.9 & 9.2 & 1. & 15.7 \\
\hline$(m|a|)^{-1}(\mathrm{~km} / \mathrm{s})$ & 205 & 250 & 15205 & 100 \\
$4 \pi a^{2} / m\left(\mathrm{~cm}^{2} / \mathrm{g}\right)$ & 1.7 & 5. & 1. & 6.3 \\
\hline
\end{tabular}

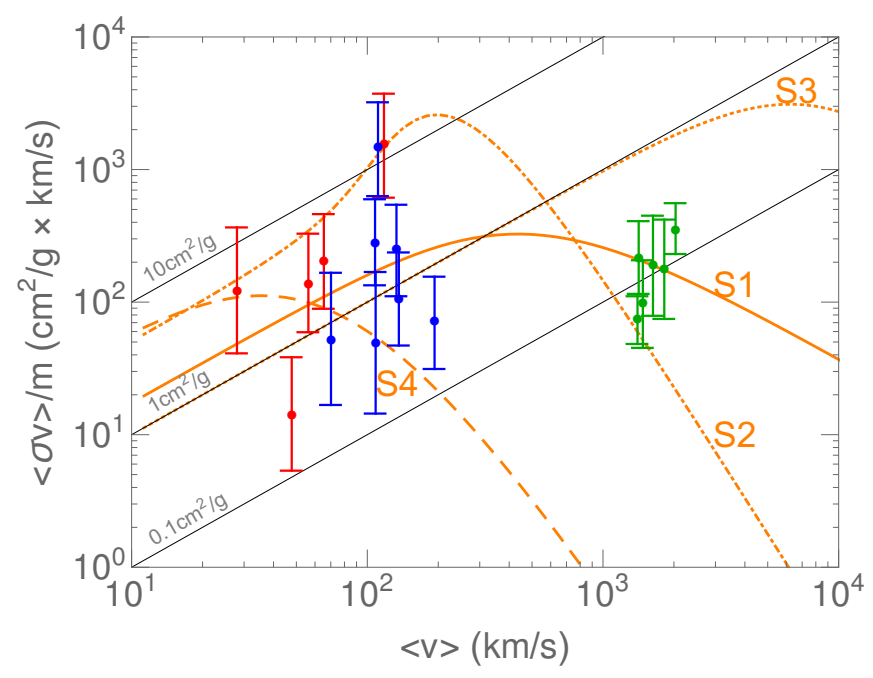

Figure 6: Fit of DM self-interaction cross sections at various astrophysical scales using effective range approach. The points gives the inferred values of $\langle\sigma v\rangle / m$ taken from [12. The curves of S1-4 show the averaged $\langle\sigma v\rangle / m$ as a function of $v$, calculated for four benchmark parameter sets (see the top table). Note that although $a$ is set to be positive in the table, changing $a \rightarrow-a$ and $r_{e} \rightarrow-r_{e}$ simultaneously results in the same curve. Among them, S1 gives the best-fit set.

Fig. 6 and corresponds to the benchmark S1. ${ }^{7}$ As expected, it fulfills the condition stated in Eqs. (9).

We would like to emphasize that the points shown in Fig. 6 should be taken with caution, as subtle effects, such as tidal stripping, still need to be further studied to understand the (sub-)halo dynamics (see e.g. [40 44 for recent discussions). In fact, the need of a sizeable DM self-interaction at cluster scales is under debate as this relies on the assumption that one can robustly infer the existence of cores in clusters of galaxies. This motivates us to also consider other possibilities which are not necessarily fitting the green points but in agreement with conservative bounds at cluster scales, $\sigma / m \lesssim 1 \mathrm{~cm}^{2} / \mathrm{g}$. These are the benchmarks S2, S3 and S4 labeled in Fig.6.

The benchmark S2 fits the dwarf and LSB data points fairly well with a relatively high cross section but is too low to fully accommodate the cluster points. This is be-

\footnotetext{
${ }^{7}$ As mentioned before, the sign of $a$ or $r_{e}$ can not be fit by studying the velocity dependence of the scattering. Moreover, since S1 has very small value of $r_{e}$, flipping the sign of either of them, in practice, gives the same cross section as a function of the velocity.
} 
cause its peak is very pronounced with $r_{e} / a \gg 1$ and $\langle\sigma v\rangle$ decreases very rapidly for $\langle v\rangle$ greater than the peak velocity. This can be achieved with a narrow resonance [20. In contrast, another benchmark S3 describes an almost constant self-interaction cross section. Interestingly, this is the benchmark that gives the lowest DM mass. As mentioned below, this is the sort of points expected in particle models with contact interaction or heavy mediators. Finally, benchmark S4 describes a velocity-averaged cross section whose peak velocity is around $50 \mathrm{~km} / \mathrm{s}$ and avoids potentially stringent bounds on self-interaction cross section from massive galaxy/cluster observations.

\section{INTERPRETING $a$ AND $r_{e}$ IN TERMS OF MODEL PARAMETERS}

In this section, we discuss how the scattering length and the effective range are related to the model parameters of several SIDM scenarios, including those with a light mediator [2, 17, resonant SIDM [20, as well as Strongly Interactive Massive Particles (SIMP) 45,47.

\section{A. Contact interaction}

The simplest model discussed for SIDM is

$$
V=\frac{1}{2} m^{2} \phi^{2}+\frac{1}{4 !} \lambda \phi^{4}
$$

where $m$ is the DM mass and $\lambda$ the coupling. It leads to a constant cross section (within the Born approximation)

$$
\sigma_{0}=\frac{\lambda^{2}}{128 \pi m^{2}} .
$$

In the effective-range framework, this happens for $\left|r_{e}\right| \ll$ $|a| \ll k^{-1}$ so that

$$
\sigma_{0}=4 \pi a^{2} .
$$

For the Born amplitude to be trusted, we need $\lambda \lesssim 1$, and hence

$$
m \lesssim 8.14 \mathrm{MeV} \lambda^{2 / 3}\left(\frac{1 \mathrm{~cm}^{2} / \mathrm{g}}{\sigma_{0} / m}\right)^{1 / 3} .
$$

If we believe in the upper limit from the clusters, such contact interaction provides a poor fit to the data. Note that the benchmark point S3 corresponds to a large coupling $\lambda \sim 10^{5}$ for $15 \mathrm{GeV} \mathrm{DM}$, where we can no longer trust the Born approximation. Typically, the $\phi^{4}$ theory requires a UV completion of a strongly-coupled dynamics among dark matter particles.

\section{B. SIDM with a light mediator}

Models in which non-relativistic DM is coupled to a boson of mass $m_{\phi}$ predict DM self-interactions mediated by the Yukawa potential of Eq. (5). Using the numerical method discussed in Appendix A, we calculate the $S$ wave scattering length and effective range together with the corresponding exact and approximated cross sections, for both repulsive and attractive cases. The results are shown in Fig. 7, Fig. 8 and Fig. 9, respectively. Notice that fixing $\alpha \mathrm{m} / \mathrm{m}_{\phi}$ and $v / \alpha$ determines all quantities in units of $m_{\phi}^{-1}$. The figure also gives the $\left(a, r_{e}\right)$ parameters of the Hulthén potential $V(r)= \pm \alpha \delta e^{-\delta r} /\left(1-e^{-\delta r}\right)$, which has been used to approximate the Yukawa potential by setting $\delta=2 \zeta(3) m_{\phi}$ 48. Both potentials give similar effective-range parameters, and thus similar selfinteracting cross sections.

a. The Born regime: In this case $\alpha m \ll m_{\phi}$ and the phase shift can be found by solving the Schrödinger equation perturbatively. In this way, according to Eq. A8, we have

$$
\begin{aligned}
\tan \delta_{0} & \simeq-m k \int_{0}^{\infty} r^{2} V(r) \frac{\sin ^{2}(k r)}{(k r)^{2}} d r \\
& =\frac{m \alpha k}{m_{\phi}^{2}}\left(1-\frac{2 k^{2}}{m_{\phi}^{2}}+\mathcal{O}\left(\frac{k^{4}}{m_{\phi}^{4}}\right)\right),
\end{aligned}
$$

which implies

$$
a=-\frac{m \alpha}{m_{\phi}^{2}}, \quad \text { and } \quad r_{e}=\frac{4}{m \alpha} .
$$

Therefore, in the limit of very small $\alpha$, the scattering length is negligible, the effective range $r_{e}$ is large and they have opposite signs. This behaviour is clearly shown in Fig. 7. As can be seen from the $k^{2}$ expansion of Eq. (15), even in this Born regime, the effective range formula $k \cot \delta_{0}=-1 / a+r_{e} k^{2} / 2$ can only approximate the $S$-wave phase shift for $k \ll m_{\phi}$. The opposite case is the classical regime mentioned before, where higher partial waves have to be taken into account.

$b$. The resonant regime: Now we turn to the parameter regime satisfying $\alpha m \gtrsim m_{\phi}$, where non-perturbative effects play an important role. In particular, the attractive case exhibits a very rich phenomenology. This is in sharp contrast to the repulsive case shown in Fig. 8 , where the scattering cross section simply increase with larger couplings.

For attractive interactions, Fig. 7 shows that as $m \alpha / m_{\phi}$ gradually increases, a critical value is reached at which the scattering length goes to negative infinity. This corresponds to a phase shift approaching $\pi / 2$ from below and a cross section of $\sigma_{0}=4 \pi / k^{2}$. Notice that this is the maximum value allowed by unitarity. Immediately after $m \alpha / m_{\phi}$ exceeds such a critical value, $a$ becomes positively infinite. 

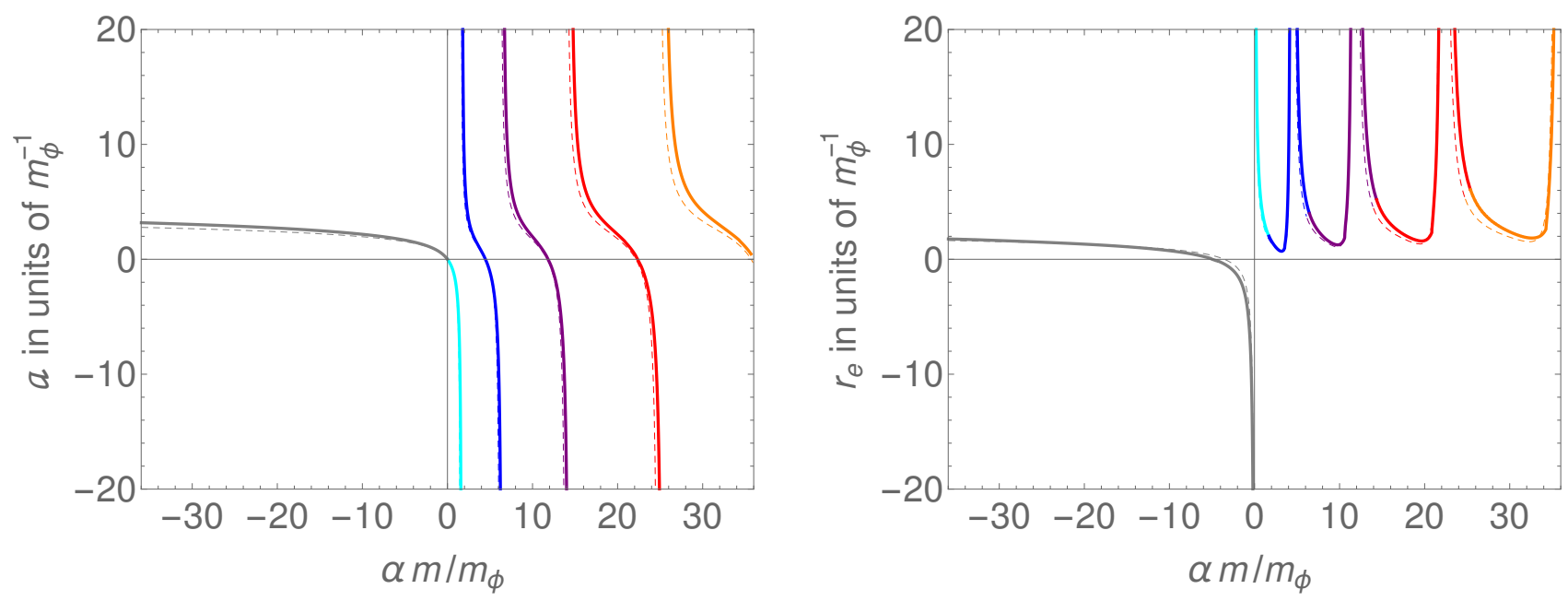

Figure 7: Left: $S$-wave scattering length as a function of $\alpha m / m_{\phi}$ for the Yukawa (solid) and the Hulthén (dashed) potentials. The case of a repulsive force $(\alpha<0)$ is shown in gray. For the attractive case $(\alpha>0)$, as $\alpha m / m_{\phi}$ increases, a different color is chosen after the phase shift reaches an odd multiple of $\pi / 2$. This indicates a parametric resonance, where the cross section reaches a maximum (the unitarity limit) and the scattering length diverges. The antiresonances, $\delta=0$, correspond to vanishing scattering lengths and thus zero cross sections. Right: Same as the left panel but for the $S$-wave effective range.

Then, with even larger $m \alpha / m_{\phi}$, the scattering length starts to decrease, until it reaches zero, corresponding to a phase shift of $\pi$. This is the so-called antiresonance, where the cross section takes its minimum value. Further increasing $m \alpha / m_{\phi}$ leads to negative values for $a$, which eventually approaches negative infinity again. The same cycle repeats itself indefinitely. Notice that this behavior of the cross section is responsible for the peak structure observed in Figs. 11 and 9. As mentioned in the previous section, there is a close connection between those peaks,

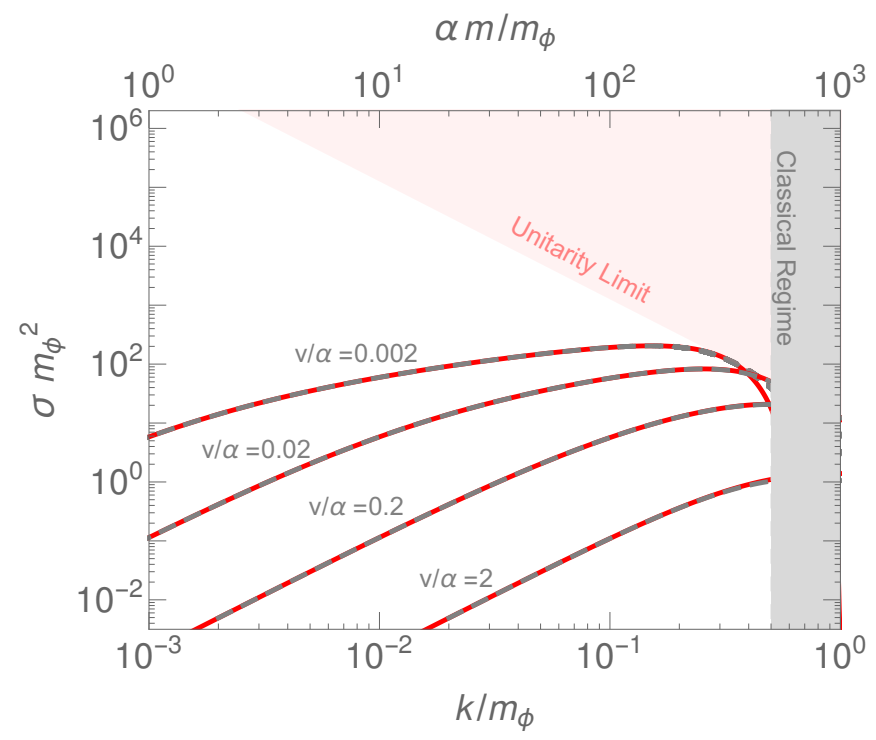

Figure 8: $S$-wave scattering cross section for the repulsive Yukawa potential in Eq. (5). The red solid lines are the numerical results, while the dashed gray lines are given by the corresponding effective-range approximation. where $|a| \rightarrow \infty$, and the bound states that are formed due to the Yukawa potential. Below, we discuss this and how they are related to the poles of the scattering amplitude.

\section{SIDM via bound states or virtual levels}

Eq. (4) has the following poles

$$
k_{ \pm}^{\text {pole }}=\frac{i}{a} \frac{2}{1 \pm \sqrt{1-2 r_{e} / a}} .
$$

Even though the poles are in general complex, they can influence the low-energy scattering if they are sufficiently close to the incoming particle momentum. In fact, a close inspection of the Schrödinger equation allows us to interpret them in terms of physical states. ${ }^{8}$ In Fig. 10 we plot the real and the imaginary parts of $k_{+}^{\text {pole }}$, which is the closer pole to the real axis.

For simplicity, let us consider first the case of a pure imaginary $k^{\text {pole }}$. The corresponding energy $E=$ $\left(k^{\text {pole }}\right)^{2} /\left(2 m_{\star}\right)$ is negative, indicating the existence of a bound state with binding energy $\epsilon=-E$. Eq. (17) then leads to

$$
2 m_{\star} \epsilon=\left(\frac{1}{a}+m_{\star} \epsilon r_{e}\right)^{2} .
$$

This formula is remarkable. Take as an example the case of proton-neutron system in the spin-1 configuration.

\footnotetext{
${ }^{8}$ For a textbook review of these topics, see Landau \& Lifshitz 49 ].
} 


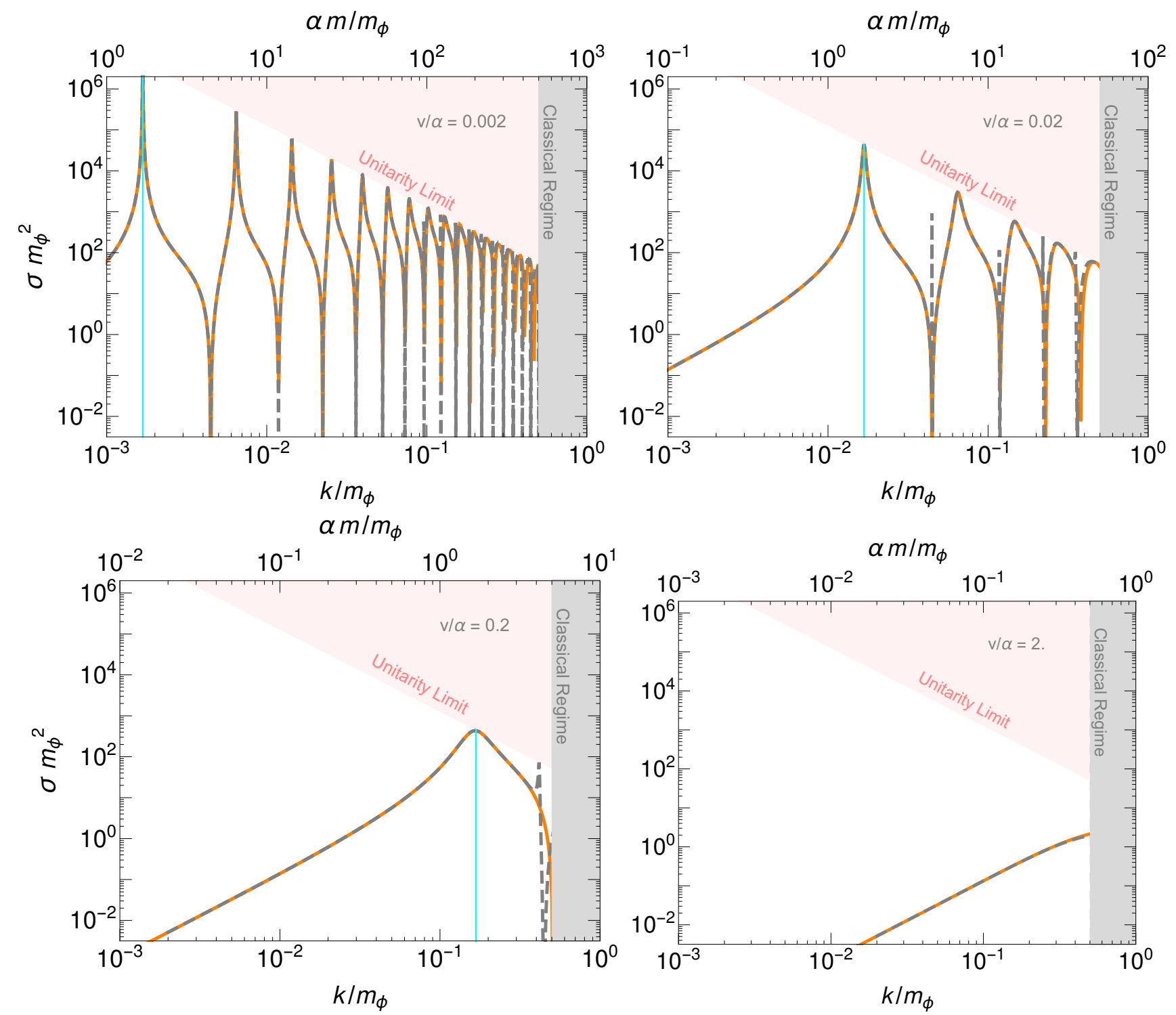

Figure 9: $S$-wave scattering cross section for the attractive Yukawa potential in Eq. (5). The orange solid lines are the numerical results, while the dashed gray lines are the corresponding effective-range approximation. Vertical cyan lines correspond to the first resonance at $\alpha m / m_{\phi} \simeq 1.68$.

The values quoted above $\left(a=5.42 \mathrm{fm}\right.$ and $\left.r_{e}=1.75 \mathrm{fm}\right)$, which characterize the velocity dependence of the cross section $\sigma_{p n}$, can be used to solve for the binding energy of the deuteron. The result is in perfect agreement with the observed value of $\epsilon=2.2 \mathrm{MeV}$.

Nonetheless, not every pole is related to a bound state. The latter are only associated with $k^{\text {pole }}=i|k|$ (i.e., $\operatorname{Im} k>0)$. Poles with a negative imaginary part correspond to either virtual levels $\left(k^{\text {pole }}=-i|k|\right)$ or resonances $\left(k^{\text {pole }}=\kappa_{d}-i\left|\gamma_{d}\right|\right) .{ }^{9}$ An example of the former

\footnotetext{
${ }^{9}$ For $\kappa_{d} \neq 0$, the imaginary part of $k^{\text {pole }}$ cannot be positive to conserve the total probability, see e.g. [50].
}

is given by the collision of neutrons $(a=-18.9 \mathrm{fm}$ and $r_{e}=2.75 \mathrm{fm}$ ). No bound state of two neutrons exists in nature. In fact, the state inducing such scattering is a virtual level.

The relevance of this for SIDM is that if DM forms a bound state, as predicted in many well-motivated scenarios, the corresponding binding energy would be related to the parameters that determine velocity dependence of the self-interaction cross section by means of Eq. (18). This is particularly true for the Yukawa potential. At the peaks of the cross section (see e.g. Fig. 1), we found that $|a| \rightarrow \infty$. Eqs. (17 18 in turn suggest $|a| \sim 1 / \sqrt{2 m \epsilon}$ with $\epsilon \rightarrow 0$. The peaks in the cross section are thus related to the existence of nearly zero-energy bound states.

Even though the regime associated with such bound 

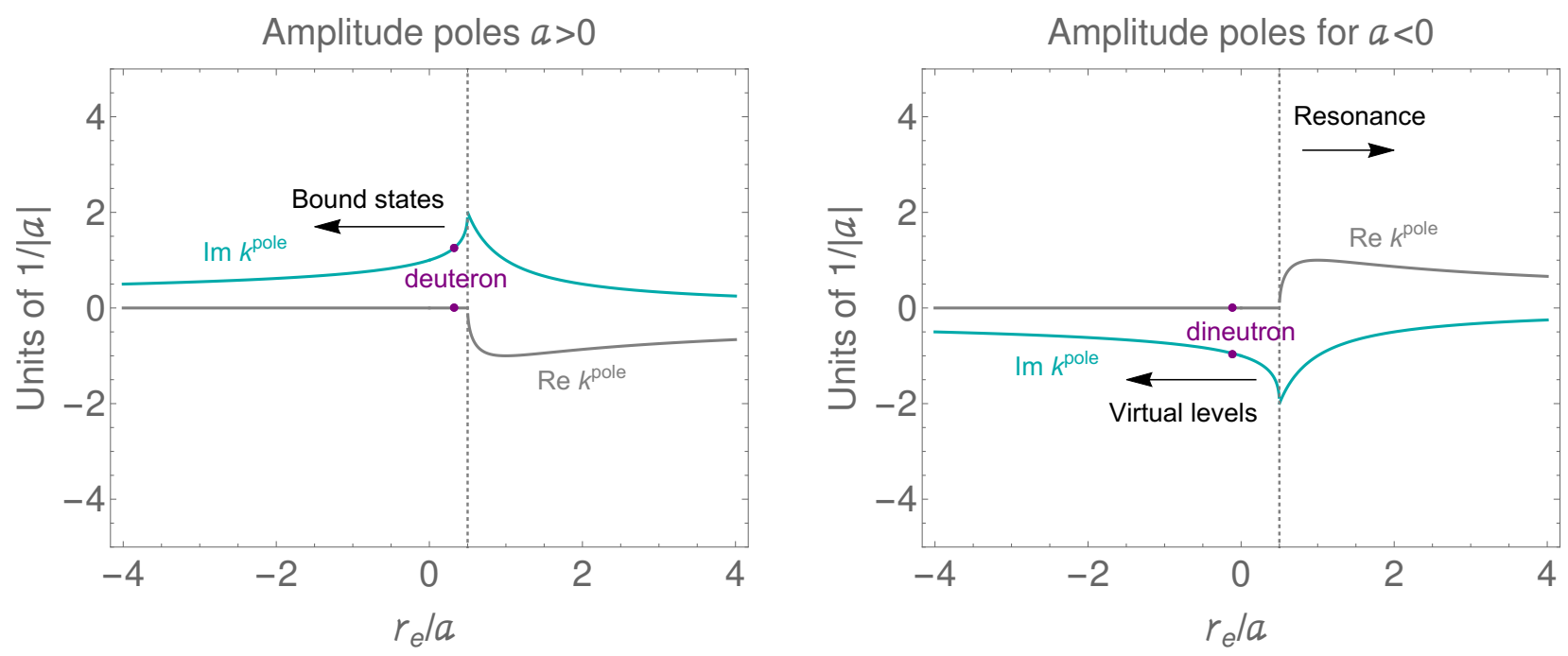

Figure 10: The real and imaginary parts of $k_{+}^{\text {pole }}$ in units of $1 /|a|$ as a function of $r_{e} / a$ for both $a>0$ (left) and $a<0$ (right). The corresponding physical states are labelled in texts. Note that in left panel the pole at $r_{e} / a>1 / 2$ is unphysical (see footnote 9.

states is usually referred to as "resonant regime", we would like to emphasize that there are no intermediate particles produced on shell, i.e., particle resonances. Instead, there are parametric resonances in the sense that for certain parameter combinations the cross section saturates the unitarity limit, where $\epsilon$ approaches zero. All this explains why the presence of poles with very small $\epsilon$ affects the scattering cross section dramatically.

\section{Resonant SIDM}

In the case of a particle resonance mediating the selfscattering, it is straightforward to see that the kinetic energy $E=\left(k^{\text {pole }}\right)^{2} /\left(2 m_{\star}\right)$ is complex, with its real and imaginary parts corresponding to the energy above the threshold $E_{R}$, and the decay width of the resonant state $\Gamma(E)$, respectively. More precisely, for the $\ell=0$ case, $E=E_{R}-i \Gamma(E) / 2$, which together with Eq. (7) leads to the well-known formulas

$$
\begin{aligned}
\delta_{0} & =\tan ^{-1}\left(\frac{\Gamma(E) / 2}{E_{R}-E}\right), \\
\sigma_{0} & =\frac{4 \pi}{m E} \frac{\Gamma(E)^{2} / 4}{\left(E-E_{R}\right)^{2}+\Gamma^{2}(E) / 4} .
\end{aligned}
$$

To conclude, when the scattering is induced by a bound state, a virtual level or a resonance, this shows up as momentum poles in the complex $k$ plane. Depending on the sign of the scattering length and the ratio $r_{e} / a$, the effective range theory allows to predict which one actually takes place. In fact, one can elaborate further on the nature of the intermediate state in the scattering process using $r_{e}$ and $a$. For instance, in the context of the deuteron, Weinberg showed that one can infer whether the intermediate state is composite or not from the sign of the effective range [51]. Discussing these interesting topics lies beyond the scope of this work.

\section{E. SIMPs}

The Strongly Interacting Massive Particle (SIMP) is a proposal where the thermal freeze-out occurs by a $3 \rightarrow 2$ transition, which is important when the dynamics is strongly coupled, hence the name 47. It can be naturally realized in QCD-like gauge theories where pions interact via the Wess-Zumino-Witten term 52. Many variations and mediation mechanisms are discussed in the literature $[53-74$.

The SIMP mechanism prefers dark matter mass in the range from $100 \mathrm{MeV}$ to $\mathrm{GeV}$, and is in marginal conflict with the cluster data as seen in Fig. 5. On the other hand, the strong dynamics often leads to existence of real resonances, bound states, and/or virtual states, which can improve the agreement by suppressing the cross section at high velocities. In fact, such resonances in QCD-like models of SIMPs are possible [75].

\section{IMPROVING THE EFFECTIVE-RANGE APPROXIMATION}

Although Figs. 8 and 9 show that the effective-range approximation works remarkably well in large portions of the parameter space of the Yukawa potential, they make clear that the approximation fails close to the parameter points where the cross section vanishes, i.e., at the antiresonances. In fact, for realistic $S$-wave Breit-Wigner resonances, it may not work for all possible values of the momentum. Likewise, so far we have used the effective 


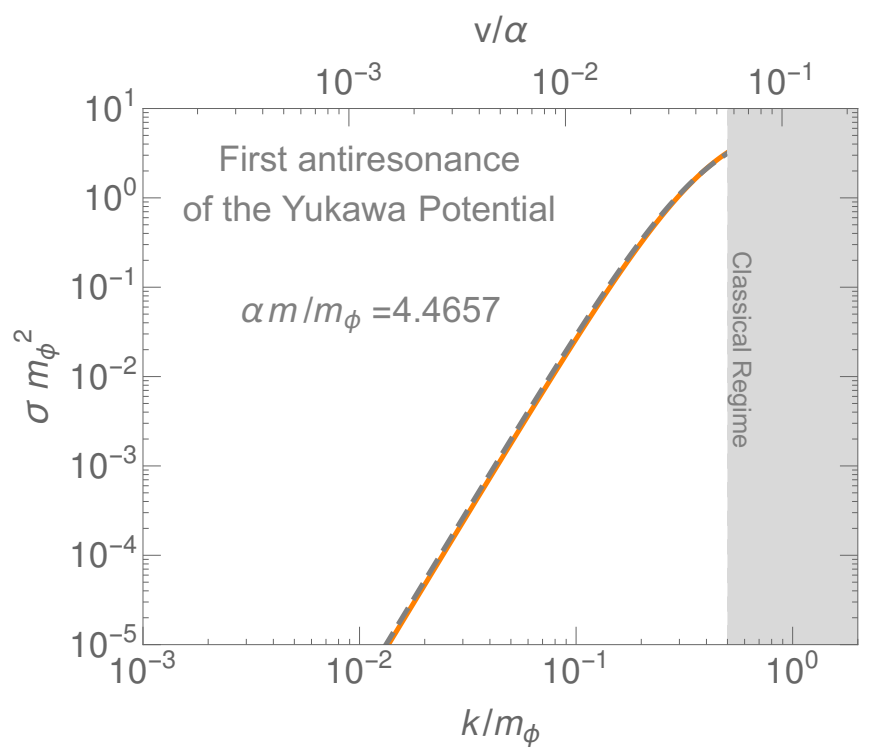

Figure 11: $S$-wave cross section for the first antiresonance of the attractive Yukawa potential (solid-orange) and the approximation (dotted-gray) based on the improved effectiverange formula (Eq. 21) ) giving $\mathfrak{a} \approx R=-0.85$ and $\mathfrak{r}_{\mathfrak{e}}=13.4$. For comparison, the standard effective-range approximation gives $a=0$ and therefore a negligible cross section everywhere, which does not show up in the plot.

range approach to discuss the non-relativistic DM scattering induced by short-range interactions while inelastic scatterings have been neglected to make sure that the potential, as well as the phase shift, is always real. In this section, we demonstrate that all these effects can be properly described by extending the effective range formalism.

\section{A. Antiresonances}

$S$-wave antiresonances are probably the simplest example where the effective range formalism fails. In contrast to the prediction of Eq. (4), the scattering amplitude and the cross section vanish at a particular value of the momentum, but not everywhere. One possible way to account for this is to decompose the total phase shift into two pieces - one of them satisfying the effective range approximation- in such a way that they interfere destructively. More precisely,

$$
e^{2 i \delta_{0}(k)}=e^{2 i k R} e^{2 i \delta_{a}(k)}=e^{2 i k R}\left(\frac{-\frac{1}{\mathfrak{a}}+\frac{1}{2} \mathfrak{r}_{\mathfrak{e}} k^{2}+i k}{-\frac{1}{\mathfrak{a}}+\frac{1}{2} \mathfrak{r}_{\mathfrak{e}} k^{2}-i k}\right),
$$

which leads to the scattering amplitude

$$
f_{0}(k)=\frac{e^{2 i \delta_{0}(k)}-1}{2 i k}=\frac{e^{2 i k R}-1}{2 i k}+\frac{e^{2 i k R}}{-\frac{1}{\mathfrak{a}}+\frac{1}{2} \mathfrak{r}_{\mathfrak{e}} k^{2}-i k},
$$

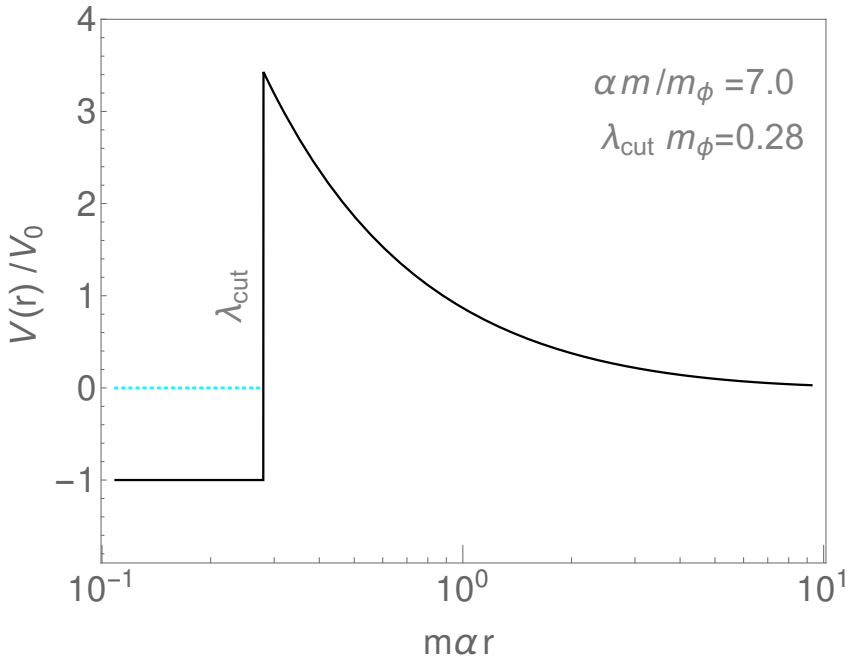

Figure 12: Potential in Eq. 23, which exhibits unstable bound-states, that is, real resonances.

vanishing at certain values of the momentum, as required. Note that here $\mathfrak{a}$ and $\mathfrak{r}_{\mathfrak{e}}$ are not the standard scattering length and effective range.

At small $k$, this is equivalent to $f_{0}(k)=R+(-1 / \mathfrak{a}+$ $\left.\mathfrak{r}_{\mathfrak{e}} k^{2} / 2-i k\right)^{-1}$, as suggested in $\S 134$ of Ref. [49. However, the latter expression does not respect unitarity because $\left|e^{2 i \delta_{0}(k)}\right| \neq 1$ as follows from Eq. (2). In contrast, our parametrization of Eq. (21) respects unitarity manifestly.

Fig. 11]illustrates our parametrization for the first antiresonance of the attractive Yukawa potential. The difference between the numerical result and the approximation based on Eq. 21) is imperceptible.

\section{B. Sharp resonances}

Eq. 21) can also describe sharp resonances (second term) accompanied with a continuum piece (first term), while the standard effective range approximation can only describe one of the two. To illustrate this fact, let us consider the potential

$$
V(r)=-m \alpha^{2} \Theta\left(\lambda_{\text {cut }}-r\right)+\Theta\left(r-\lambda_{\text {cut }}\right) \frac{\alpha}{r} e^{-m_{\phi} r},
$$

which we depict in Fig. 12 for a particular parameter choice.

This potential gives rise to positive-energy bound states that decay through quantum tunneling. These are real resonances in contrast to the peaks associated with the Yukawa potential in Fig. 1, as explained above. In fact, as shown in the left panel of Fig. 13, the scattering cross section exhibits a resonant enhancement for certain values of the momentum. These correspond to the formation of unstable bound-states. Interestingly, while the standard effective range formula describes the continuum 

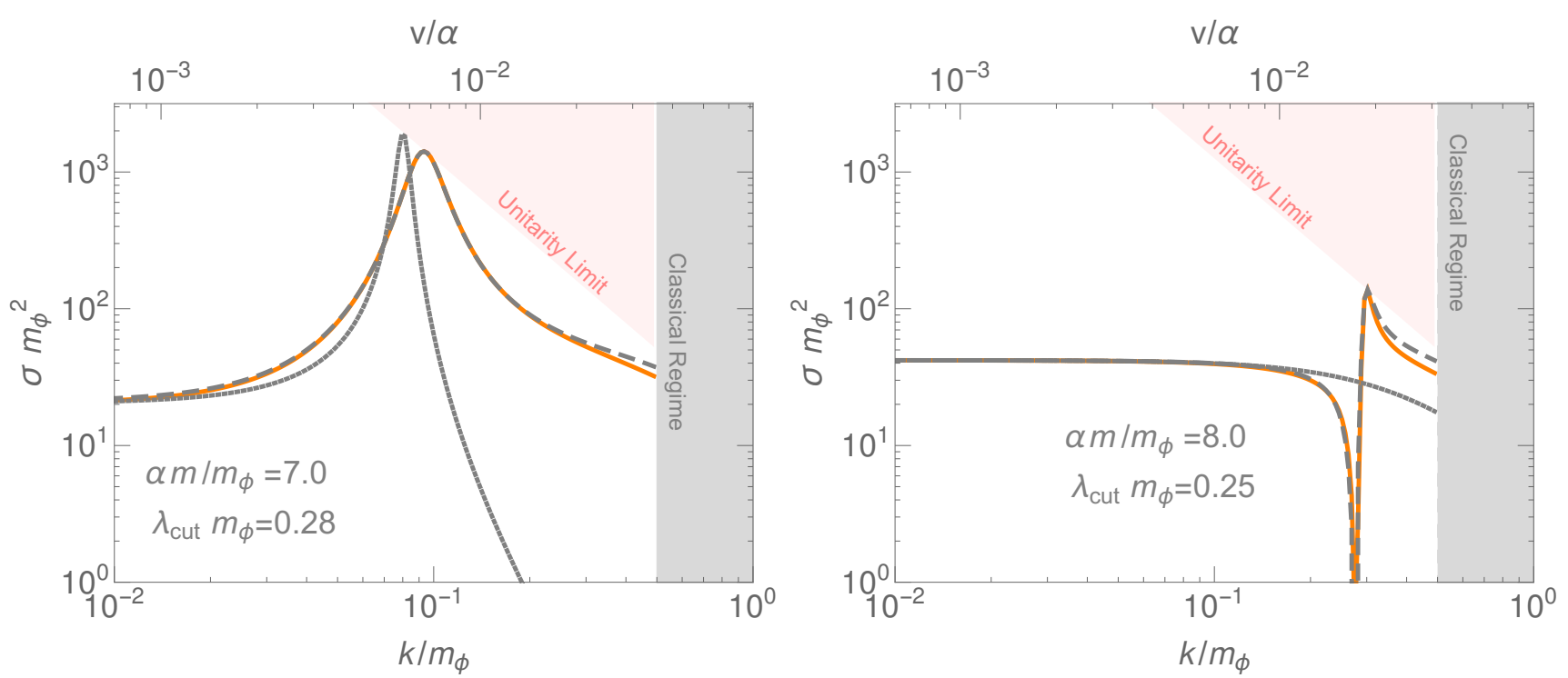

Figure 13: Same as Fig. 11 but for the potential of Eq. (23). The exact result is the solid orange line, the dashed line is the approximation based on Eq. 21) while the dotted gray line is the standard effective-range approximation of Eq. (4).

part of cross section fairly well, it fails to describe scattering cross section at the peak. In spite of this, the improved formula approximates the exact result very well. Notice that, as explained above, such an improvement is in practice adding a continuum piece to cross section (except for negative and positive interference just below and above the resonance), which has already been considered in phenomenological studies of resonant SIDM [20].

In the same fashion, Eq. (21) can simultaneously describe a resonance and an antiresonace as it is the case for potential in Eq. (23) for certain points of the parameter space. This is shown in the right panel of Fig. 13 for another parameter choice. In this case, the antiresonance is induced by the destructive inferences between the continuum and resonance parts.

\section{Inelastic scatterings}

Even though we have assumed that inelastic scatterings - such as DM annihilation or radiative capture [23, 24 - are subleading, in principle they can play a role. For instance, in the vanilla light-mediator model, DM typically annihilates into the mediators, leading to a complex potential [76. In this case the corresponding phase shift can be decomposed as $\delta_{\ell}=\operatorname{Re} \delta_{\ell}+i \operatorname{Im} \delta_{\ell}$. Assuming $\left|\delta_{\ell}\right| \gg \operatorname{Im} \delta_{\ell} \geq 0$, one can adopt the $S$-wave effective range approximation in the following way

$$
k \cot \delta_{0} \simeq k \cot \left(\operatorname{Re} \delta_{0}\right)-\frac{i k \operatorname{Im} \delta_{0}}{\sin ^{2}\left(\operatorname{Re} \delta_{0}\right)} \simeq-\frac{1}{a}+\frac{r_{e}}{2} k^{2},
$$

where $a$ and $r_{e}$ contain a subleading imaginary component 77. If we further neglect the imaginary component of $r_{e}$, the $S$-wave annihilation and scattering cross sec- tions are related via

$$
\sigma_{a n, 0}(k)=\frac{4 \pi}{k^{2}} \frac{1-\left|e^{2 i \delta_{0}}\right|^{2}}{4} \simeq \frac{\sigma_{0}(k)}{k} \frac{|\operatorname{Im} a|}{(\operatorname{Re} a)^{2}} .
$$

Note that such expression does not violate the unitarity limit as its last factor vanishes at $|a| \rightarrow \infty$. Moreover, it shows that $\sigma_{a n, 0}(k)$ becomes constant at $k \ll 1 /|a|$ as long as the effective range approximation applies. For more discussions on parametrizing the relation between elastic and inelastic cross sections, see e.g. [24, 76.

\section{SUMMARY AND OUTLOOK}

In this work, we have studied the effective range approach as a model-independent way to parametrize DM scattering cross sections in astrophysical halos. While it only contains two parameters besides the DM mass, it provides a good description of the self-scattering in most appealing SIDM scenarios, including SIMPs, SIDM with a light mediator and resonant SIDM models.

Starting with a brief introduction to the effective-range approach, we have studied the astrophysical implications. In general, there exists a velocity scale, $(m|a|)^{-1}$, below which the scattering cross section can be treated as a constant. For velocities well above this scale, the cross section quickly decreases. Taking bounds derived from current cluster observations, we have reached the conclusion that DM masses below several $\mathrm{GeV}$ are excluded for $\sigma / m \sim 10 \mathrm{~cm}^{2} / \mathrm{g}$ in dwarf-sized halos. See Figs. 4 and 5. The tentative non-vanishing values of $\sigma / m$-extracted from observational data at various scales- can also be fit in terms of the effective-range parameters. See Fig. 6 . Our results suggest that more precise measurements and 
better extractions are needed to identify or constrain such parameters, especially $r_{e}$.

In addition, we have further investigated the correspondence of the scattering length $a$ and the effective range $r_{e}$ to the model parameters of several popular SIDM scenarios. In general, the scattering cross sections calculated from $a$ and $r_{e}$ agrees well with the exact values as long as the range of the interaction is sufficiently short. Moreover, the effective-range approach demonstrates that significant enhancements in the selfscattering cross section are induced by the poles in the complex plane of the DM momentum. In analogy to nuclear physics, such poles can be interpreted as intermediate physical states, such as a bound state, a virtual level or a resonance.

In the end, we have briefly commented on possible extensions of the effective-range approach, especially for the cases that contain anti-resonances or sharp resonances. Besides, we have also shown that it is possible to study subleading inelastic processes such as DM annihilations using the same framework.

We believe the effective-range approach provides a simple, yet very useful, parametrization to consistently take into account the velocity dependence of DM selfinteractions in cosmological simulations involving different astrophysical scales. For instance, such velocitydependence may play an important role in better understanding the evolution of the sub-halos that move inside the Milky Way halo. This is left for future work.

\section{Acknowledgements}

We thank Kai Schmidt-Hoberg for discussions. X.C. is supported by the 'New Frontiers' program of the Austrian Academy of Sciences. C.G.C. is supported by the ERC Starting Grant NewAve (638528). X.C. and C.G.C. thank the Erwin Schrödinger International Institute for hospitality while this work was completed. H.M. thanks the Alexander von Humboldt Foundation for support while this work was completed. H.M. was supported by the NSF grant PHY-1638509, by the U.S. DOE Contract DE-AC02-05CH11231, by the JSPS Grant-in-Aid for Scientific Research (C) (17K05409), MEXT Grant-in-Aid for Scientific Research on Innovative Areas (15H05887, 15K21733), by WPI, MEXT, Japan, by the Binational Science Foundation (grant No. 2016153), and by Hamamatsu Photonics K.K.

\section{Appendix A: The effective range theory}

The phase shifts associated with the self-scattering of DM particles are obtained by solving the Schrödinger equation for the radial wavefunction $R_{\ell, k}(r)$ of the re- duced DM two-particle system. This is given by

$$
\frac{1}{r^{2}} \frac{d}{d r}\left(r^{2} \frac{d R_{\ell, k}}{d r}\right)+\left(k^{2}-\frac{\ell(\ell+1)}{r^{2}}-m V(r)\right) R_{\ell, k}=0,
$$

together with a boundary condition demanding that $r R_{l, k}$ must vanish at $r=0$. In fact, close to the origin it is expected that the angular-momentum term dominates for sufficiently well-behaved potentials, in which case $R_{l, k} \propto r^{l}$. At large distances from the origin, the potential vanishes and the wave function must be that of a free particle, i.e., a superposition of two spherical waves. The phase shift, $\delta_{\ell}$, parametrizes such a superposition. More precisely, at $r \rightarrow \infty$ the asymptotic behavior $R_{\ell, k}(r)$ is given by

$R_{\ell, k}(r) \propto \cos \delta_{\ell} j_{\ell}(k r)-\sin \delta_{\ell} n_{\ell}(k r) \approx \frac{1}{r} \sin \left(k r-\frac{l \pi}{2}+\delta_{\ell}\right)$,

where $j_{\ell}$ and $n_{\ell}$ are respectively the spherical Bessel functions of first and second order.

\section{A simple method to find the phase shift}

In the SIDM context, Ref. 35 presented a systematic method for solving Eq. A1. Here we would like to point out a simpler possibility that will not only provide a powerful method to solve for the phase shift but will also allow us to define the scattering length and the effective range. Let us first define

$$
t_{\ell, k}(r)=\frac{j_{\ell}(k r)\left(\frac{R_{\ell, k}^{\prime}(r)}{R_{\ell, k}(r)}-\frac{\ell}{r}\right)+k j_{\ell+1}(k r)}{n_{\ell}(k r)\left(\frac{R_{\ell, k}^{\prime}(r)}{R_{\ell, k}(r)}-\frac{\ell}{r}\right)+k n_{\ell+1}(k r)} .
$$

Simple algebra shows that

$$
\frac{d t_{\ell, k}(r)}{d r}=-k m r^{2} V(r)\left(j_{\ell}(k r)-t_{\ell, k}(r) n_{\ell}(k r)\right)^{2} .
$$

The fact that $R_{\ell, k} \propto r^{\ell}$ and Eq. A2 fix the boundary conditions of this differential equation to

$$
t_{\ell, k}(0)=0 \quad \text { and } \quad t_{\ell, k}(r) \rightarrow \tan \delta_{\ell} \quad \text { at } \quad r \rightarrow \infty
$$

Notice that $j_{\ell}(k r) \propto k^{\ell}$ and $n_{\ell}(k r) \propto k^{-(\ell+1)}$ in the limit $k \rightarrow 0$, which together with Eq. A4 imply that $\tan \delta_{\ell} \propto k^{2 \ell+1}$ for small momenta. The corresponding coefficient of proportionality defines scattering length $a_{\ell}$. More precisely,

$$
a_{\ell}^{2 \ell+1} \equiv-\lim _{k \rightarrow 0} \frac{\tan \delta_{\ell}}{k^{2 \ell+1}} .
$$

The function $k^{2 \ell+1} \cot \delta_{\ell}$ is thus analytic at $k=0$. The next-to-leading term determines the effective range, $r_{e, \ell}$, 
by means of

$$
k^{2 \ell+1} \cot \delta_{\ell}=-\frac{1}{a_{\ell}^{2 \ell+1}}+\frac{1}{2 r_{e, \ell}^{2 \ell-1}} k^{2}+\mathcal{O}\left(k^{4}\right) .
$$

As a by-product we have found a powerful method to solve for the phase shift. In fact, it is numerically much more efficient to integrate Eq. (A4) than to integrate Eq. (A1), not only because the former is of first order but also because solving Eq. (A4) does not require matching the solution to a plane wave at infinity in order to find the phase shift.

Eq. (A4) can be solved by expanding on the potential, with the first term determining the Born regime, which is given by

$$
\left.\tan \delta_{l}\right|_{\text {Born }}=-k m \int_{0}^{\infty} r^{2} V(r) j_{\ell}(k r)^{2} d r .
$$

\section{The $S$-wave case}

Let us take $\ell=0$ and introduce $u_{k}(r)=r R_{k, 0}(r)$. Then, Eqs. A1) and (A2) read

$$
\left(\frac{d^{2}}{d r^{2}}+k^{2}-m V(r)\right) u_{k}(r)=0,
$$

and

$$
u_{k}(0)=0, u_{k}(r) \rightarrow \psi_{k}(r)=\frac{\sin \left(k r+\delta_{0}\right)}{\sin \delta_{0}} \text { at } r \rightarrow \infty .
$$

Here we have chosen a convenient normalization factor for $u_{k}$. In the following we will find it useful to employ the previous definition of $\psi_{k}(r)$ for any positive value of $r$. Simple algebra proves that for any potential

$$
u_{k}(r) \frac{d u_{0}(r)}{d r}-\left.u_{0}(r) \frac{d u_{k}(r)}{d r}\right|_{0} ^{r}=k^{2} \int_{0}^{r} u_{0}\left(r^{\prime}\right) u_{k}\left(r^{\prime}\right) d r^{\prime} .
$$

Moreover, using the fact that $\psi_{k}(r)$ is the solution of the Schrödinger equation for $V(r)=0$, we find that

$$
\psi_{k}\left(r^{\prime}\right) \frac{d \psi_{0}\left(r^{\prime}\right)}{d r}-\left.\psi_{0}\left(r^{\prime}\right) \frac{d \psi_{k}\left(r^{\prime}\right)}{d r}\right|_{0} ^{r}=k^{2} \int_{0}^{r} \psi_{0}\left(r^{\prime}\right) \psi_{k}\left(r^{\prime}\right) d r^{\prime}
$$

Notice that $\psi_{0}(r)=1-r / a_{0}$, where $a_{0}$ is the scattering length. Subtracting Eq. A11 from Eq. A12, taking $r \rightarrow \infty$ and using the fact that $u_{k}$ and $\psi_{k}$ approach to each other in that limit, we find that

$$
\begin{aligned}
k \cot \delta_{0} & =-\frac{1}{a_{0}}+k^{2} \int_{0}^{\infty}\left(\psi_{0} \psi_{k}-u_{0} u_{k}\right) d r \\
& =-\frac{1}{a_{0}}+\frac{1}{2} r_{e, 0} k^{2}+\mathcal{O}\left(k^{4}\right) .
\end{aligned}
$$

where

$$
r_{e, 0}=2 \int_{0}^{\infty}\left(\psi_{0}^{2}-u_{0}^{2}\right) d r .
$$

This is the original expression found by Bethe [28, who argued that the expansion in Eq. A13 approximates the phase shift with a great accuracy because $\psi_{k}$ and $u_{k}$ differ only where the potential is non-negligible. Note that this conclusion is based on the assumption that in this region both wave functions depend very weakly on $k$, which is generally true, when the potential energy is much larger than kinetic energy and $k r$ is small.

To qualitatively understand the effective range, one can consider the following upper bound, which is valid for potentials that effectively vanish at distances greater than certain range $R[78$, as it is the case of the Yukawa potential. Then, $\psi_{0}$ and $u_{0}$ in Eq. A14 coincide for $r \gtrsim R$, which implies that

$$
\begin{aligned}
\frac{r_{e, 0}}{R} & \approx \frac{2}{R} \int_{0}^{R}\left(\left(1-\frac{r}{a_{0}}\right)^{2}-u_{0}^{2}\right) d r \\
& \leq 2\left(1-\frac{R}{a_{0}}+\frac{1}{3}\left(\frac{R}{a_{0}}\right)^{2}\right) .
\end{aligned}
$$

Moreover, for shallow attractive potentials, $u_{0}$ behaves like a slowly-varying sine function, where mostly $u_{0} \lesssim \psi_{0}$, resulting in a positive $r_{e, 0}$. See also $\S .133$ of [49].

\section{The Hulthén Potential}

In the main text, it has been mentioned that the Hulthén potential

$$
V(r)= \pm \alpha \delta e^{-\delta r} /\left(1-e^{-\delta r}\right)
$$

approximates well the Yukawa potential if one sets $\delta=$ $2 \zeta(3) m_{\phi}$, where $\alpha$ gives the coupling and $m_{\phi}$ is the mediator mass of the Yukawa potential [48. The advantage of employing the Hulthén potential is that its corresponding Schrödinger equation is analytically solvable, and yield the $S$-wave phase shift 35,48 .

$$
\delta_{0}=\arg \left(\frac{i \Gamma\left(\lambda_{+}+\lambda_{-}-2\right)}{\Gamma\left(\lambda_{+}\right) \Gamma\left(\lambda_{-}\right)}\right) .
$$

Here, the dimensionless function $\lambda_{ \pm}$is given by $1+$ $i m v /(2 \delta) \pm \sqrt{\alpha m / \delta-m^{2} v^{2} /(2 \delta)^{2}}$.

Using Eqs. A13) and A14, one can obtain the analyt- 


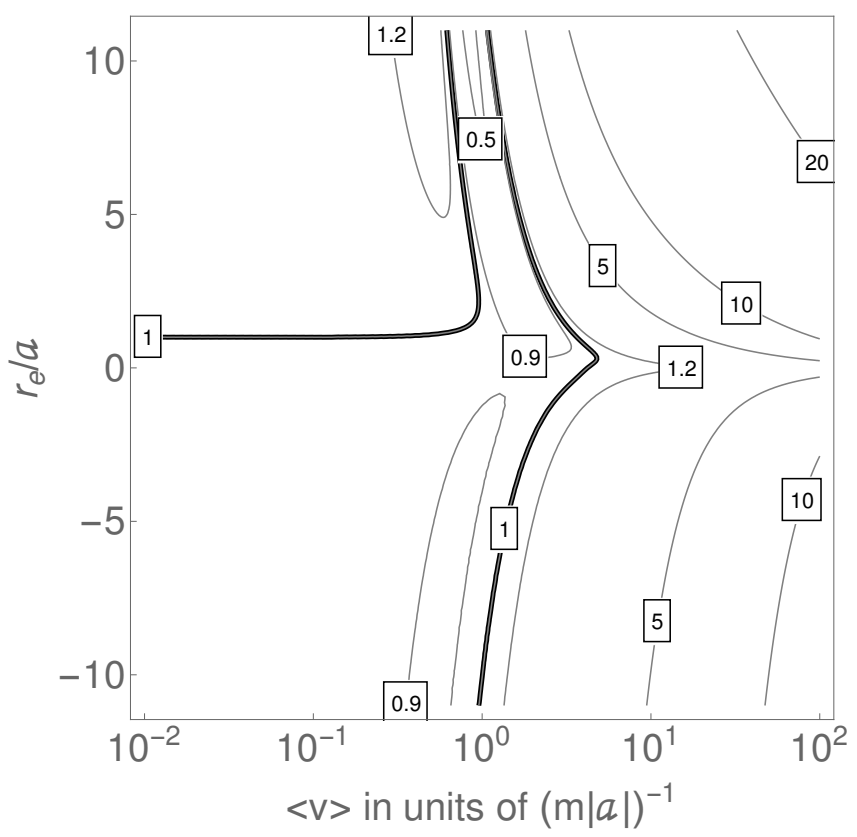

Figure 14: Contours of the ratio of $\langle\sigma v\rangle$ and $\sigma(\langle v\rangle)\langle v\rangle$ as a function of $a m\langle v\rangle$ and $r_{e} / a$.

ical expressions of the $S$-wave effective-range parameters as

$$
\begin{aligned}
a= & \frac{\psi^{(0)}(1+\eta)+\psi^{(0)}(1-\eta)+2 \gamma}{\delta} \\
r_{e}= & \frac{2 a}{3}-\frac{1}{3 \delta \eta\left[\psi^{(0)}(1+\eta)+\psi^{(0)}(1-\eta)+2 \gamma\right]^{2}} \\
& \times\left\{3\left[\psi^{(1)}(1+\eta)-\psi^{(1)}(1-\eta)\right]\right. \\
& \left.+\eta\left[\psi^{(2)}(1+\eta)+\psi^{(2)}(1-\eta)+16 \zeta(3)\right]\right\}
\end{aligned}
$$

where $\eta=\sqrt{\alpha m / \delta}, \psi^{(n)}(z)$ are the polygamma functions of order $n$ and $\gamma \simeq 0.5772$ is the Euler-Mascheroni constant.

\section{Appendix B: Velocity-averaged cross sections}

The averaged cross section $\langle\sigma v\rangle$ can be calculated in terms of

$$
\begin{aligned}
\frac{\langle\sigma v\rangle}{\langle v\rangle} & \equiv \frac{\int_{0}^{\infty} f\left(v, v_{0}\right) \sigma v d v}{\int_{0}^{\infty} f\left(v, v_{0}\right) v d v} \\
& =\pi a^{2} \frac{r_{e}^{2}\left(z_{+}-z_{-}\right)}{a^{2}-2 a r_{e}}\left(\phi\left(z_{+}\right)-\phi\left(z_{-}\right)\right),
\end{aligned}
$$

with

$$
\begin{aligned}
\phi(z) & =z e^{-z} \Gamma(0,-z) \\
z_{ \pm} & =\frac{32}{\pi(a m\langle v\rangle)^{2}}\left(1-\frac{a}{r_{e}} \pm \frac{a}{r_{e}} \sqrt{1-\frac{2 r_{e}}{a}}\right) \frac{a}{r_{e}}
\end{aligned}
$$

We also show the ratio of $\langle\sigma v\rangle$ and $\sigma(\langle v\rangle)\langle v\rangle$ as a function of $a m\langle v\rangle$ and $r_{e} / a$ in Fig. 14, which shows that within the effective-range approach both coincide at one percent level, except for the large-velocity regime $\langle v\rangle \gg(|a| m)^{-1}$, where $\sigma$ can be sensitive to $v$.
[1] N. Aghanim et al. (Planck) (2018), 1807.06209.

[2] D. N. Spergel and P. J. Steinhardt, Phys. Rev. Lett. 84, 3760 (2000), astro-ph/9909386.

[3] S. Tulin and H.-B. Yu, Phys. Rept. 730, 1 (2018), 1705.02358.

[4] J. S. Bullock and M. Boylan-Kolchin, Ann. Rev. Astron. Astrophys. 55, 343 (2017), 1707.04256.

[5] R. Dave, D. N. Spergel, P. J. Steinhardt, and B. D. Wandelt, Astrophys. J. 547, 574 (2001), astro-ph/0006218.

[6] M. Vogelsberger, J. Zavala, and A. Loeb, Mon. Not. Roy. Astron. Soc. 423, 3740 (2012), 1201.5892.

[7] M. Rocha, A. H. G. Peter, J. S. Bullock, M. Kaplinghat, S. Garrison-Kimmel, J. Onorbe, and L. A. Moustakas, Mon. Not. Roy. Astron. Soc. 430, 81 (2013), 1208.3025.

[8] A. H. G. Peter, M. Rocha, J. S. Bullock, and M. Kaplinghat, Mon. Not. Roy. Astron. Soc. 430, 105 (2013), 1208.3026 .

[9] O. D. Elbert, J. S. Bullock, S. Garrison-Kimmel, M. Rocha, J. Oorbe, and A. H. G. Peter, Mon. Not. Roy.
Astron. Soc. 453, 29 (2015), 1412.1477.

[10] A. B. Fry, F. Governato, A. Pontzen, T. Quinn, M. Tremmel, L. Anderson, H. Menon, A. M. Brooks, and J. Wadsley, Mon. Not. Roy. Astron. Soc. 452, 1468 (2015), 1501.00497.

[11] S. W. Randall, M. Markevitch, D. Clowe, A. H. Gonzalez, and M. Bradac, Astrophys. J. 679, 1173 (2008), 0704.0261.

[12] M. Kaplinghat, S. Tulin, and H.-B. Yu, Phys. Rev. Lett. 116, 041302 (2016), 1508.03339.

[13] A. Robertson, R. Massey, and V. Eke, Mon. Not. Roy. Astron. Soc. 465, 569 (2017), 1605.04307.

[14] K. Bondarenko, A. Boyarsky, T. Bringmann, and A. Sokolenko, JCAP 1804, 049 (2018), 1712.06602.

[15] O. D. Elbert, J. S. Bullock, M. Kaplinghat, S. GarrisonKimmel, A. S. Graus, and M. Rocha, ApJ 853, 109 (2018), 1609.08626.

[16] D. Harvey, A. Robertson, R. Massey, and I. G. McCarthy (2018), 1812.06981. 
[17] J. L. Feng, M. Kaplinghat, and H.-B. Yu, Phys. Rev. Lett. 104, 151301 (2010), 0911.0422.

[18] M. R. Buckley and P. J. Fox, Phys. Rev. D81, 083522 (2010), 0911.3898.

[19] S. Tulin, H.-B. Yu, and K. M. Zurek, Phys. Rev. Lett. 110, 111301 (2013), 1210.0900.

[20] X. Chu, C. Garcia-Cely, and H. Murayama, Phys. Rev. Lett. 122, 071103 (2019), 1810.04709.

[21] M. Ibe and H.-b. Yu, Phys. Lett. B692, 70 (2010), 0912.5425 .

[22] M. Duch and B. Grzadkowski, JHEP 09, 159 (2017), 1705.10777.

[23] J. D. March-Russell and S. M. West, Phys. Lett. B676, 133 (2009), 0812.0559.

[24] E. Braaten and H. W. Hammer, Phys. Rev. D88, 063511 (2013), 1303.4682.

[25] J. M. Cline, Z. Liu, G. Moore, and W. Xue, Phys. Rev. D90, 015023 (2014), 1312.3325.

[26] E. Braaten, D. Kang, and R. Laha, JHEP 11, 084 (2018), 1806.00609 .

[27] R. Mahbubani, M. Redi, and A. Tesi (2019), 1908.00538.

[28] H. A. Bethe, Phys. Rev. 76, 38 (1949).

[29] J. M. Blatt and J. D. Jackson, Phys. Rev. 76, 18 (1949).

[30] H. P. Noyes, Ann. Rev. Nucl. Part. Sci. 22, 465 (1972).

[31] V. G. J. Stoks, R. A. M. Klomp, M. C. M. Rentmeester, and J. J. de Swart, Phys. Rev. C48, 792 (1993).

[32] T. F. O'Malley, L. Spruch, and L. Rosenberg, Journal of Mathematical Physics 2, 491 (1961).

[33] R. O. Berger and L. Spruch, Phys. Rev. 138, B1106 (1965).

[34] H. van Haeringen and L. P. Kok, Phys. Rev. A26, 1218 (1982).

[35] S. Tulin, H.-B. Yu, and K. M. Zurek, Phys. Rev. D87, 115007 (2013), 1302.3898.

[36] A. B. Newman, T. Treu, R. S. Ellis, and D. J. Sand, Astrophys. J. 765, 25 (2013), 1209.1392.

[37] R. Kuzio de Naray, S. S. McGaugh, and W. J. G. de Blok, Astrophys. J. 676, 920 (2008), 0712.0860.

[38] S.-H. Oh, W. J. G. de Blok, E. Brinks, F. Walter, and R. C. Kennicutt, Jr, Astron. J. 141, 193 (2011), 1011.0899 .

[39] M. Valli and H.-B. Yu, Nat. Astron. 2, 907 (2018), 1711.03502 .

[40] A. Sokolenko, K. Bondarenko, T. Brinckmann, J. Zavala, M. Vogelsberger, T. Bringmann, and A. Boyarsky, JCAP 1812, 038 (2018), 1806.11539.

[41] J. Kummer, M. Brggen, K. Dolag, F. Kahlhoefer, and K. Schmidt-Hoberg (2019), 1902.02330.

[42] M. Kaplinghat, M. Valli, and H.-B. Yu (2019), 1904.04939.

[43] O. Sameie, H.-B. Yu, L. V. Sales, M. Vogelsberger, and J. Zavala (2019), 1904.07872.

[44] F. Kahlhoefer, M. Kaplinghat, T. R. Slatyer, and C.-L. Wu (2019), 1904.10539.

[45] A. D. Dolgov, Yad. Fiz. 31, 1522 (1980).

[46] E. D. Carlson, M. E. Machacek, and L. J. Hall, Astrophys. J. 398, 43 (1992).

[47] Y. Hochberg, E. Kuflik, T. Volansky, and J. G. Wacker, Phys. Rev. Lett. 113, 171301 (2014), 1402.5143.

[48] S. Cassel, J. Phys. G37, 105009 (2010), 0903.5307.

[49] L. D. Landau and E. M. Lifshits, Quantum Mechanics, vol. v.3 of Course of Theoretical Physics (Butterworth-
Heinemann, Oxford, 1991), ISBN 9780750635394.

[50] A. G. Sitenko and P. J. Shepherd, Lectures in scattering theory, Internat. Ser. Mono. Natural Philos. (Pergamon, Oxford, 1971), trans. from the Russian, URL https:// cds.cern.ch/record/102667.

[51] S. Weinberg, Phys. Rev. 137, B672 (1965).

[52] Y. Hochberg, E. Kuflik, H. Murayama, T. Volansky, and J. G. Wacker, Phys. Rev. Lett. 115, 021301 (2015), 1411.3727.

[53] N. Yamanaka, S. Fujibayashi, S. Gongyo, and H. Iida (2014), 1411.2172.

[54] H. M. Lee and M.-S. Seo, Phys. Lett. B748, 316 (2015), 1504.00745

[55] M. Hansen, K. Langæble, and F. Sannino, Phys. Rev. D92, 075036 (2015), 1507.01590.

[56] N. Bernal and X. Chu, JCAP 1601, 006 (2016), 1510.08527.

[57] N. Bernal, C. Garcia-Cely, and R. Rosenfeld, JCAP 1504, 012 (2015), 1501.01973.

[58] N. Bernal, X. Chu, C. Garcia-Cely, T. Hambye, and B. Zaldivar, JCAP 1603, 018 (2016), 1510.08063.

[59] S.-M. Choi and H. M. Lee, JHEP 09, 063 (2015), 1505.00960 .

[60] Y. Hochberg, E. Kuflik, and H. Murayama, JHEP 05, 090 (2016), 1512.07917.

[61] E. Kuflik, M. Perelstein, N. R.-L. Lorier, and Y.-D. Tsai, Phys. Rev. Lett. 116, 221302 (2016), 1512.04545.

[62] S.-M. Choi and H. M. Lee, Phys. Lett. B758, 47 (2016), 1601.03566 .

[63] S.-M. Choi, Y.-J. Kang, and H. M. Lee, JHEP 12, 099 (2016), 1610.04748.

[64] D. Pappadopulo, J. T. Ruderman, and G. Trevisan, Phys. Rev. D94, 035005 (2016), 1602.04219.

[65] M. Farina, D. Pappadopulo, J. T. Ruderman, and G. Trevisan, JHEP 12, 039 (2016), 1607.03108.

[66] U. K. Dey, T. N. Maity, and T. S. Ray (2016), 1612.09074

[67] J. Cline, H. Liu, T. Slatyer, and W. Xue (2017), 1702.07716 .

[68] S.-M. Choi, H. M. Lee, and M.-S. Seo, JHEP 04, 154 (2017), 1702.07860.

[69] S.-M. Choi, Y. Hochberg, E. Kuflik, H. M. Lee, Y. Mambrini, H. Murayama, and M. Pierre (2017), 1707.01434.

[70] X. Chu and C. Garcia-Cely, Phys. Rev. D96, 103519 (2017), 1708.06764.

[71] S.-M. Choi, H. M. Lee, P. Ko, and A. Natale, Phys. Rev. D98, 015034 (2018), 1801.07726.

[72] A. Berlin, N. Blinov, S. Gori, P. Schuster, and N. Toro, Phys. Rev. D97, 055033 (2018), 1801.05805.

[73] S.-M. Choi, H. M. Lee, Y. Mambrini, and M. Pierre (2019), 1904.04109.

[74] S. Bhattacharya, P. Ghosh, and S. Verma (2019), 1904.07562.

[75] R. McGehee, H. Murayama, and Y.-D. Tsai, in preparation (2019).

[76] K. Blum, R. Sato, and T. R. Slatyer, JCAP 1606, 021 (2016), 1603.01383.

[77] J. Mitroy, Phys. Rev. A 66, 022716 (2002).

[78] D. R. Phillips and T. D. Cohen, Phys. Lett. B390, 7 (1997), nucl-th/9607048. 